\title{
A target expression threshold dictates invader defense and autoimmunity by CRISPR-Cas13
}

\author{
Elena Vialetto ${ }^{1}$, Yanying Yu ${ }^{1}$, Scott P. Collins ${ }^{2}$, Katharina G. Wandera ${ }^{1}$, Lars Barquist ${ }^{1,3}$, Chase \\ L. Beisel $\left.\right|^{1,2,3 *}$
}

${ }^{1}$ Helmholtz Institute for RNA-based Infection Research (HIRI), Helmholtz Centre for Infection Research (HZI), 97080 Würzburg, Germany

2Department of Chemical and Biomolecular Engineering, North Carolina State University, Raleigh, NC 27695, USA

${ }^{3}$ Medical Faculty, University of Würzburg, 97080 Würzburg, Germany

*Correspondence to: chase.beisel@helmholtz-hiri.de (to C.L.B.)

\section{HIGHLIGHTS}

- Cas13-induced dormancy requires RNA target levels to exceed an expression threshold

- The expression threshold can prevent cytotoxic self-targeting for endogenous transcripts

- The threshold shifts depending on the CRISPR RNA guide:target pair

- The threshold allows cells to distinguish pathogenic and benign infections 


\section{SUMMARY}

2 Immune systems must recognize and clear foreign invaders without eliciting autoimmunity.

3 CRISPR-Cas immune systems in prokaryotes manage this task by following two criteria:

4 extensive guide:target complementarity and a defined target-flanking motif. Here we report an

5 additional requirement for RNA-targeting CRISPR-Cas13 systems: expression of the target

6 transcript exceeding a threshold. This finding is based on targeting endogenous non-essential

7 transcripts, which rarely elicited dormancy through collateral RNA degradation. Instead, eliciting

8 dormancy required over-expressing targeted transcripts above a threshold. A genome-wide

9 screen confirmed target expression levels as the principal determinant of cytotoxic autoimmunity

10 and revealed that the threshold shifts with the guide:target pair. This expression threshold

11 ensured defense against a lytic bacteriophage yet allowed tolerance of a targeted beneficial gene

12 expressed from an invading plasmid. These findings establish target expression levels as a third

13 criterion for immune activation by RNA-targeting CRISPR-Cas systems, buffering against

14 autoimmunity and distinguishing pathogenic and benign invaders. 


\section{INTRODUCTION}

All cellular immune systems face the challenge of differentiating foreign entities from the host. Failure to recognize features associated with an invader exposes the host to a potentially fatal infection, while failure to ignore similar features in the host can trigger a catastrophic autoimmune response. Elucidating how immune systems make these decisions and the consequences of errors is critical not only for treating infectious and autoimmune diseases in higher eukaryotes but also for understanding the physiology and evolution of single-cell microbes under constant assault by mobile genetic elements and bacteriophages (Goldberg and Marraffini, 2015; Hampton et al., 2020; Theofilopoulos et al., 2017).

CRISPR-Cas systems, as the only known adaptive immune systems in bacteria and archaea, face these same challenges (Barrangou et al., 2007; Makarova et al., 2015, 2020). These systems store fragments of invading genetic material as spacers situated between conserved repeats in CRISPR arrays (Barrangou et al., 2007; Jackson et al., 2017). To stem an infection, the arrays are transcribed and processed into individual CRISPR RNAs (crRNAs) that direct the system's effector nucleases to complementary genetic sequences (Charpentier et al., 2015; Hille et al., 2018; Marraffini and Sontheimer, 2010; van der Oost et al., 2014). The nature of the target genetic material (i.e., DNA or RNA) and the consequence of recognizing a target sequence vary widely across systems. For example, Type II CRISPR-Cas systems recognize target sequences within double-stranded DNA flanked by a protospacer-adjacent motif (PAM)

34 (Leenay and Beisel, 2017), which triggers the Cas9 effector nuclease to create a clean DNA cut (Gasiunas et al., 2012; Jinek et al., 2012). Separately, Type VI CRISPR-Cas systems recognize target sequences within RNA lacking complementarity between the flanking sequence and

37 repeat-derived "tag" within the crRNA (also called a protospacer-flanking sequence or PFS)

38 (Meeske and Marraffini, 2018; Wang et al., 2021). Target recognition by the Type VI Cas13 39 effector nuclease activates non-specific cleavage of cellular RNAs (Abudayyeh et al., 2016; East-

40 Seletsky et al., 2016). Widespread RNA degradation induces growth arrest--a state called cellular 
41 dormancy--that prevents the replication and dissemination of the invader (Abudayyeh et al., 2016;

42 Meeske et al., 2019).

Whether for RNA-targeting or DNA-targeting CRISPR-Cas systems, immune defense is

44 activated in the presence of a nucleic acid complementary to the crRNA guide and flanked by an

45 appropriate sequence. The requirement for target complementarity allows the nuclease to ignore

46 similar but not identical sequences potentially present in the cell. The requirement for the flanking

47 sequence helps the nuclease distinguish the target sequence of the invader from the

48 corresponding spacer within the CRISPR array, as they contain the same complementary

49 sequence but different flanking sequences. However, in the infrequent instance of a spacer being

50 acquired from a chromosomal sequence, this pairing would be expected to drive cytotoxic

51 autoimmunity, killing the cell or driving either mutation of the target or inactivation of the CRISPR-

52 Cas system (Rollie et al., 2020; Stern et al., 2010).

Here, we report that Type VI CRISPR-Cas systems encoding Cas13 nucleases take into

54 account a third criterion for immune activation: minimal expression of the target transcript. This expression threshold is higher than most cellular transcripts under our experimental conditions,

56 allowing the co-existence of a targeted endogenous transcript and an active immune system. The

57 threshold allows the immune system to tolerate a passive invader such as a plasmid yet mount a 58 robust immune response to an actively replicating invader such as a lytic phage. These insights 59 support a model for the impact of Cas13 activation on the cell, where transcripts below the 60 threshold are tolerated or lead to specific target cleavage and transcripts above the threshold lead 61 to widespread RNA degradation. This model may help explain why applying Cas 13 in eukaryotes

62 yields programmable gene silencing in some contexts (Abudayyeh et al., 2017; Cox et al., 2017)

63 and non-specific RNA degradation in others (Buchman et al., 2020; Konermann et al., 2018;

64 Wang et al., 2019; Xu et al., 2021).

65

\section{RESULTS}


68 Cas13-induced immunity has been principally assessed through targeting transcripts expressed

69 from plasmids or phages (Abudayyeh et al., 2016, 2017; Kiga et al., 2020; Meeske and Marraffini, 70 2018; Meeske et al., 2019). What has remained less clear is the impact of targeting 71 chromosomally-encoded transcripts, particularly given that targeting chromosomally-encoded

72 transcripts in eukaryotes has been largely associated with targeted gene silencing (Abudayyeh et 73 al., 2017; Cox et al., 2017). To address this gap, we employed the Cas13 nuclease from the Type

74 VI-A CRISPR-Cas system in Leptotrichia shahii (LshCas13a) (Abudayyeh et al., 2016; Liu et al.,

75 2017a; Watanabe et al., 2019) in Escherichia coli as a simple model of immune defense,

76 paralleling prior studies showing that this nuclease could drive collateral RNA cleavage and

77 dormancy (Abudayyeh et al., 2016; East-Seletsky et al., 2016; Liu et al., 2017b). We first verified

78 Cas 13a activity by targeting either of two different sites within a synthetic transcript constitutively

79 expressed from a plasmid, which resulted in a 2,800-fold and 460-fold reduction in the

80 transformation efficiency of the respective crRNA-encoding plasmid versus a non-targeting

81 crRNA plasmid (Fig. 1A-B). To verify that the reduction in transformation depended on Cas13a

82 endonuclease activity, we inactivated the HEPN domain of the nuclease using a previously

83 characterized point mutation (R1278A) that abolishes RNA degradation (Abudayyeh et al., 2016).

84 The resulting catalytically-dead Cas13a (dCas13a) yielded similar colony counts between

85 targeting and non-targeting crRNAs, demonstrating that Cas13a RNase activity is responsible

86 for the observed reduction in plasmid transformation (Fig. 1B). We further confirmed that targeting

87 the synthetic transcript induces collateral activity based on the degradation of total RNA upon 88 target expression (Fig. S1).

After validating the targeting activity of LshCas13a in E. coli, we proceeded to assess self-

90 targeting. Previous studies of Cas13a in bacteria suggested that targeting leads to dormancy as

91 long as the target transcript is present (Abudayyeh et al., 2016; Meeske and Marraffini, 2018), so

92 we expected to observe autoimmunity for all the targets expressed under standard growth 
conditions. To assess self-targeting, we designed 12 crRNAs targeting mRNAs encoded by essential and non-essential genes in E. coli following current guide design rules (Wessels et al., 2020) and repeated the transformation assay (Fig. 1A). Surprisingly, targeting yielded a negligible reduction in colony counts for all but two targets: one in the essential gapA mRNA and another in the non-essential yfaP mRNA (Fig. 1C). Repeating the assay with dCas13a or without the nuclease revealed that the transformation reduction with the yfaP-targeting crRNA was due to cytotoxicity associated with the crRNA itself (Figs. 1C and S2). While some Cas13a crRNAs are known to exhibit poor targeting activity (Wessels et al., 2020), the chance that we had selected almost entirely "bad" guides seemed low. Therefore, other factors may be necessary to explain the consistent lack of reduced transformation.

\section{Boosting target expression above a threshold enables Cas13-based immunity}

Given that transcript levels are generally lower when expressed from the chromosome versus a multicopy plasmid, we asked if levels of the target transcript play a role in the induction of Cas 13 immunity. We constitutively expressed a 72-nucleotide (nt) fragment of selected mRNA targets on a plasmid (Fig. 2A). Expressing these fragments without disrupting the endogenous locus resulted in a 280 -fold to 1,000 -fold reduction in colony counts compared to the non-targeting control, indicating that target expression levels impact the immune response. The large reduction

111 in colony counts was lost when using dCas13a (Fig. 2A), confirming the involvement of RNA cleavage.

Our observations suggested that a certain target expression level might need to be

114 reached to elicit the immune response. To explore this possibility, we expressed the plasmid115 encoded synthetic transcript targeted at two locations $(\mathrm{T} 1, \mathrm{~T} 2)$ under eight different constitutive 116 promoters (P1 to P8) (Table S1) exhibiting varying expression strengths (Fig. 2B-C). While the 117 three weakest promoters yielded no reduction in transformation efficiency compared to the non118 targeting control, we observed a strong reduction for the remaining promoters $(\sim 1,100$-fold for T1, 
60-fold for T2). No reduction was observed with dCas13a for any of the promoters (Fig. S3).

120 The transition between low and high transformation efficiencies was remarkably sharp and

121 occurred for both targets between the same two promoters separated by only a 5.8-fold difference

122 in transcriptional activity (Fig. 2C). We performed a similar analysis with the full-length soxS

123 mRNA using the same set of constitutive promoters (Fig. 2D), where the transformation reduction

124 occured only with the strongest promoter. These results show that low target expression

125 prevented immune induction, while boosting target expression beyond a threshold was sufficient

126 to induce Cas13-based immunity.

A genome-wide screen establishes target expression levels as the principal determinant of immune induction

130 Thus far, our observations were based on a small number of endogenous targets. In order to 131 determine to what extent expression thresholds influence targeting across the transcriptome and 132 whether other factors (e.g., target position, sequence context) also impact the outcome of 133 targeting, we performed a genome-wide screen. We designed a library of 25,597 crRNAs 134 targeting all chromosomally-encoded mRNAs and rRNAs in E. coli (Fig. 3A). The guide sequences were selected following current design rules (e.g., PFS lacking complementarity to crRNA repeat tag) (Meeske and Marraffini, 2018; Wessels et al., 2020) to reduce the inclusion of

137 low-efficiency guides, and the targets were spaced across the entire length of each coding region 138 (or transcribed region in the case of rRNAs). The library included 400 randomized guides as non139 targeting controls lacking complementarity to any endogenous transcripts. We then transformed 140 the crRNA library into E. coli cells with or without LshCas13a and cultured the transformed cells 141 with antibiotic selection to deplete guides causing collateral activity and dormancy. Short-read 142 sequencing was finally applied to measure the depletion of each guide compared to the no143 LshCash13a control cultured under the same conditions (Fig. 3B). Under this setup, highly active 
144 guides would be heavily depleted within the library, while poorly active guides would be minimally 145 depleted. Initial analysis revealed that the extent of depletion depended on whether the target was

147 associated with an essential or non-essential gene. This observation is in line with the idea that 148 target transcript cleavage of an essential gene would be expected to cause a fitness defect even 149 in the absence of widespread collateral cleavage. Indeed, compared to the set of randomized 150 guides, $85 \%$ of guides targeting essential genes were depleted versus only $43 \%$ of guides 151 targeting non-essential genes. The median depletion was also higher for guides targeting 152 essential (2.9-fold) versus non-essential (0.3-fold) genes (Fig. 3C). The screen was validated by 153 testing individual highly depleted guides using the transformation assay (Fig. S4). The limited 154 depletion of guides targeting non-essential genes further shows that guides, even when designed 155 following standard rules, infrequently lead to widespread collateral cleavage and dormancy. We also noticed that guides targeting rRNAs, the highest expressed RNAs in the cell, 157 were strongly and consistently depleted in the library (Fig. 3C). To analyze in more detail how 158 transcript levels contributed to guide depletion across the library, we performed transcriptomics 159 analyses of $E$. coli cells not expressing LshCas13a and cultured in middle $\left(\mathrm{ABS}_{600} \approx 0.5\right)$ and late

$160 \quad\left(\mathrm{ABS}_{600} \approx 0.8\right)$ exponential growth phases. By correlating the resulting transcript levels with the

161 median depletion of guides targeting each gene, we found a clear correlation that was stronger 162 for transcript levels in middle exponential growth (Spearman coefficient $=0.72$ ) than for late 163 exponential growth (Spearman coefficient $=0.68)$ (Figs. 3D and S5A). The stronger correlation 164 with early exponential growth might be because this growth phase dominates the screen. 165 Translational strength predicted using the ribosome-binding site (RBS) calculator showed minimal 166 correlation (Salis, 2011) (Fig. S5B), indicating that protection of the mRNA by translating 167 ribosomes does not account for differences in guide depletion. 
In order to determine which features of the guide:target pair most impact induction of cytotoxic immunity, we subjected the dataset to machine learning (see Methods) (Fig. 3E). The resulting SHAP values (Lundberg et al., 2020) revealed that transcript levels were the strongest

171 predictor of guide depletion. In contrast, gene essentiality had a modest effect on the predicted 172 depletion for the average guide, which can be attributed to the fact that there are only a small

173 number of essential genes in E. coli. Factors related to the sequence and predicted structure of

174 the crRNA guide and RNA target also had predictive value (Figs. 3E and S6), in line with previous

175 observations from high-throughput assays of Cas13a guides (Wessels et al., 2020). Together,

176 our results demonstrate that most self-targeting guides do not activate a Cas13-based immune

177 response, at least under our experimental conditions, but that high transcript levels can trigger

178 immunity.

\section{The target expression threshold depends on the selected guide}

Although transcript levels are an important predictor of immune activation, guide features also impact the response. For example, depletion of the highly expressed tolB transcript (Fig. 4A), which was targeted by nine different guides within the library, varied between minimal depletion to depletion paralleling that of some guides targeting rRNAs (Figs. 3C and 4B). We tested each guide individually in the transformation assay and observed a strong correlation between the reduction in colony counts and the depletion score from the screen (Spearman coefficient = 0.87). Furthermore, some of the guides yielded an insignificant reduction in colony counts compared to the non-targeting control, underscoring the variability in immune activation for this one highly expressed mRNA depending on the guide sequence.

Our initial data also suggested that boosting expression of the tolB mRNA might be able to rescue immune activation for poorly performing guides. We therefore placed the tolB RBS and 192 coding region under a medium or strong constitutive promoter on a plasmid, and we repeated the 193 transformation assay (Fig. 4B). For all guides that displayed low efficiency when targeting the 
194 endogenous transcript, expressing tolB from the plasmid led to full immune activation. Notably,

195 guides that exhibited some reduction in colony counts required only the medium promoter to

196 maximize immune activation, while guides exhibiting negligible reduction in colony counts

197 required the strong promoter to achieve full immunity. Therefore, boosting target expression can

198 rescue immune activation by otherwise poorly active crRNAs. Concurrently, the target expression

199 threshold required for immune activation by Cas13a can vary between guides, even when

200 targeting the same transcript.

\section{Reducing Cas13a levels precludes immune induction}

203 We demonstrated that the expression level of the target must exceed a threshold for Cas13a to 204 induce dormancy. While thus far we focused on the properties of the target, the concentration of 205 the Cas13a:crRNA complex could also be an influencing factor. We therefore explored the impact 206 of altering levels of this complex on plasmid transformation. Specifically, we swapped the native 207 promoter from L. shahii driving transcription of cas13a with a constitutive synthetic promoter with weaker expression (Pw). After replacing cas13a with deGFP and measuring fluorescence of the cells, we measured a 44-fold decrease in fluorescence for Pw compared to the native promoter

210 (Fig. S7A). We then performed the transformation assay targeting the synthetic transcript 211 expressed from the set of constitutive promoters. Remarkably, we did not observe immune 212 activation even when expressing the synthetic target from the strongest promoter (Fig. S7B). To confirm that the nuclease is expressed, we introduced plasmids encoding the nuclease,

214 targeting or non-targeting crRNA and a deGFP reporter to measure collateral cleavage activity in 215 cell-free transcription-translation (TXTL) reactions (Liao et al., 2019; Marshall et al., 2018, 2020)

216 (Fig. S7C). Monitoring reporter fluorescence over time, we found that the nuclease expressed 217 from the Pw promoter inhibited deGFP expression compared to the non-targeting control, albeit 218 with slower kinetics than the construct with the native promoter. Nevertheless, these data indicate 219 that Cas13a expressed under the Pw promoter is active. Overall, these results show that low 
220 levels of Cas13a nuclease can impair immune activation, even when target expression levels are

221 high.

222

223 Infection by a lytic phage activates Cas13a-based immunity even for less efficient guides

224 Beyond self-targeting, another important question is how the target expression threshold impacts

225 immune activation by foreign invaders targeted by Cas13. We began with the lytic MS2 RNA

226 bacteriophage that rapidly replicates and lyses E. coli as part of the infection cycle. We designed

2276 crRNA guides (M1-M6) targeting within the rep and $c p$ genes (Fig. 5A), as both genes are non-

228 toxic when expressed in E. coli and thus can be expressed individually to determine the

229 expression threshold for each crRNA. To determine each crRNA's expression threshold for

230 immune induction, we expressed rep or cp under a weak, medium, and strong promoter on a low-

231 copy plasmid and measured the extent of plasmid interference (Fig. 5B). We found that the

232 crRNAs were associated with a wide range of target expression thresholds for immune induction,

233 with one exhibiting a low expression threshold, three exhibiting a higher expression threshold,

234 and two exhibiting an expression threshold higher than the strongest promoter.

235 We then infected E. coli cultures with MS2 phages at different MOls $(0.1,5)$ and assessed

236 defense through each of the six crRNAs (Fig. 5C). In the absence of MS2 phage, all cultures

237 exhibited continual growth over the time course. Cultures with LshCas13a and crRNAs exhibiting

238 at least some immunity in the transformation assay (M1-M4) halted growth and maintained

239 turbidity, indicative of immunity-induced dormancy. In contrast, cultures with LshCas13a and the

240 crRNAs that did not exhibit immunity in the transformation assay (M5, M6) as well as all

241 dLshCas13a controls showed a temporary drop in turbidity, indicative of successful phage

242 infection. Therefore, even crRNAs requiring high target expression to activate widespread

243 immunity can confer robust defense against a pathogenic invader. 

host

247 Beyond pathogenic invaders, we considered benign invaders that do not rapidly replicate and 248 instead generally maintain transcript levels. We reasoned that the target transcript would not elicit 249 an immune response if expressed below the expression threshold, allowing the invader to persist.

250 One notable scenario is a targeted gene that benefits the host cell, such as a virulence factor or 251 an antibiotic resistance marker spread through mobile genetic elements (Frost et al., 2005; Koonin 252 et al., 2020; Partridge et al., 2018). If targeting does not substantially affect levels of this transcript, 253 then the host could benefit from its expression but not elicit an immune response.

To explore this scenario directly, we created a plasmid encoding two resistance genes: a 255 kanamycin resistance gene targeted by two distinct crRNAs (our targeted beneficial gene) as well as a non-targeted hygromycin resistance gene (for plasmid selection). The kanamycin gene was placed downstream of a strong, medium, or weak constitutive promoter (Fig. 6A). We then assessed tolerance to and growth benefits from the kanamycin resistance gene in two separate steps: assessing tolerance by transforming the plasmid under hygromycin selection, and assessing the growth benefit by measuring growth of tolerant cells in the presence of kanamycin. For the first step, high target expression induced an immune response while low target expression was tolerated (Fig. 6B), in line with other target transcripts expressed from plasmids (Figs. 2, 4). For the second step, we found that the tolerated crRNA:promoter combinations

264 yielded substantial growth on $10 \mu \mathrm{g} / \mathrm{mL}$ kanamycin (Fig. 6C). One combination (K2 crRNA with 265 P5-expressed target) maintained growth even in the presence of $50 \mu \mathrm{g} / \mathrm{mL}$ kanamycin (Fig. S8). 266 In contrast, a plasmid lacking the kanamycin resistance gene did not yield any growth. For some 267 combinations, growth on kanamycin was slower with the targeting versus non-targeting crRNA. 268 As this same growth defect was not observed in the presence of hygromycin, the growth defect 269 on kanamycin may be attributed to Cas13a-mediated gene silencing that sensitized the cells to 
270 kanamycin. Overall, these results show that the target expression threshold can allow tolerance

271 of a benign invader, even allowing the targeted gene to provide benefits to the host cell.

\section{DISCUSSION}

274 Immune activation by CRISPR nucleases normally requires two established criteria:

275 complementarity between the crRNA guide and the target, and a PAM or PFS flanking the target

276 (Leenay and Beisel, 2017). Here, we show that the expression levels of the target transcript

277 represent a third criterion determining immune activation by the RNA-targeting nuclease Cas 13.

278 The outcome depends on whether expression levels are above or below a specific threshold (Fig.

279 7). When target transcript levels are above the threshold, widespread collateral RNA cleavage

280 leads to cell dormancy. When target transcript levels are below the threshold, cells escape

281 dormancy. In that case, target expression can be either unperturbed or silenced. Gene silencing

282 under the expression threshold is supported by two lines of evidence: extensive depletion of

283 guides targeting essential genes but not non-essential genes in the library screen (Fig. 3C) and

284 reduced growth on kanamycin but not hygromycin when targeting the $\mathrm{kan}^{R}$ gene (Figs. 6C and

285 S8). In both cases, silencing of the essential gene would reduce growth, even if the cells do not

286 enter dormancy through collateral RNA cleavage. The target expression threshold also can vary

287 between guide:target pairs, even when the targets are present in the same transcript. With

288 sufficient expression of the target transcript, however, the threshold can be crossed to activate

289 immunity.

290 One simple biochemical explanation for the threshold is the level of activated

291 Cas13:crRNA complexes. Higher levels would lead to widespread RNA cleavage that induces

292 growth arrest and dormancy. Lower levels might lead to preferential cleavage of the target,

293 resulting in specific silencing. For less efficient targets, the target transcript may be poorly

294 recognized for multiple reasons associated with guide selection (e.g., GC content, crRNA or target

295 folding) (Wessels et al., 2020), resulting in a negligible impact on target transcript levels. In some 
cases, we observed a sharp threshold, where immunity was fully activated with as little as a 5.8fold change in promoter activity driving target expression. While the extent of collateral cleavage should scale with the concentration of activated Cas13:crRNA complexes, collateral cleavage could create a positive feedback loop. Specifically, degradation of key transcripts might reduce

300 growth, leading to slowed dilution of activated Cas13:crRNA complexes (Elowitz et al., 2002),

301 leading to further RNA degradation and growth arrest. This mechanism is supported by our

302 findings that lowering Cas13a levels prevents immune induction (Fig. S7), where the 303 concentration of activated Cas13a:crRNA complexes could not be achieved to initiate the 304 feedback loop. However, there could also be other possible explanations for the sharp threshold, 305 such as cooperativity between Cas13 enzymes or how well activated complexes can diffuse 306 through the dense cytoplasm of a bacterial cell. Future work could evaluate the link between 307 collateral RNA cleavage and growth arrest as well as other factors that affect the threshold, such as expression levels of the crRNAs, the impact of multiplexed targeting, and the extent of collateral activity exhibited by different Cas13 nucleases (Abudayyeh et al., 2017; Xu et al., 2021).

311 and invader defense (Fig. 7). For targets above the threshold, immunity resembles that associated with other CRISPR-Cas systems: cytotoxicity incompatible with cell survival when targeting the host's own genetic material, or invader clearance or induced dormancy when

314 targeting an invader. For targets below the threshold, immunity takes a different form. For self315 targeting, targets under the threshold are tolerated, allowing the cells to persist with a limited 316 impact on fitness. In the event the target transcript undergoes silencing, Cas13 could serve a 317 regulatory role without compromising its ability to conduct immune defense, similar to scaRNAs 318 and Cas9 (Ratner et al., 2019). For invader defense, the threshold ties immune activation to the 319 threat level posed by the invader. For rapidly replicating invaders such as lytic phages that pose 320 a major threat, target transcripts will accumulate in the cell, crossing the threshold and induce a 321 robust immune response. For invaders such as lysogenic phages or mobile plasmids that take up 
residence in the cell and thus pose a minor threat, target transcripts may be expressed at a sufficiently low level to not induce the immune response. If targeting leads to gene silencing of essential transcripts, it could block invader replication, subsequently clearing the invader while sparing the infected cell from dormancy. In this setup, induced dormancy would instead represent a back-up strategy if the target transcript continues to accumulate in the cell.

For invader defense, one ramification of the target expression threshold is that an Acr

(Bondy-Denomy et al., 2013; Marino et al., 2020) that reduces the concentration of activated Cas13a complexes could rapidly interfere with immune activation. Accordingly, recent work reporting one of the first Acrs against Cas13a showed that the invading Acr-encoding phage could shut down CRISPR defenses and proliferate in the first wave of infection (Meeske et al., 2020). In contrast, Acrs that block DNA-targeting systems could allow only a second wave of infecting phages to proliferate, as the defenses would clear the first wave before the Acrs could fully inhibit the CRISPR defenses (Borges et al., 2018; Landsberger et al., 2018). The prior work on the Cas13a Acr attributed escape by the Acr-encoding phage to Cas13a degrading the phage transcripts but not the phage DNA, allowing the phage to eventually recover. However, we found that lowering the concentration of activated Cas13a complexes could allow phage transcripts to cross the original expression threshold without inducing dormancy. Therefore, both mechanisms could be at work for phages encoding Cas13 Acrs. RNA cleavage that could also be impacted by a target expression threshold (Kazlauskiene et al., 2017; Niewoehner et al., 2017; Rostøl and Marraffini, 2019). For these systems, collateral RNA

343 cleavage is induced through Cas10 within the activated effector complex synthesizing cyclic oligo 344 adenylates (cOAs) from ATP. These small molecules then bind to different accessory proteins 345 such as Csm6/Csx1 encoded by some Type III CRISPR-Cas systems, which then begin non346 specifically cleaving cellular RNAs. A key difference compared to Cas13 is that one activated 347 Type III effector complex can produce a large number of cOA molecules, potentially activating a 
348 large number of accessory proteins. This intermediate amplification step could effectively 349 eliminate the threshold, leading to widespread collateral RNA cleavage and dormancy. However, 350 depending on how quickly the cOAs are synthesized, diffuse, and turnover, a minimal threshold 351 of activated effector complexes may be necessary to elicit widespread RNA cleavage.

352 Furthermore, the existence of cOA-degrading anti-CRISPR proteins could offer a means to 353 artificially raise the target expression threshold to the point where this mode of immunity is never 354 activated over the course of the infection. Similar behaviors may be expected for Type VI 355 CRISPR-Cas systems encoding Cas13b and the accessory protein Csx28 that appears to amplify 356 the immune response (Smargon et al., 2017; VanderWal et al., 2021). Exploring the extent to 357 which a target expression threshold exists for different Type III and VI-B CRISPR-Cas systems 358 could broaden our understanding of the target expression threshold, particularly when back-up defenses exist that enhance the immune response.

Finally, the target expression threshold may help resolve otherwise contradictory observations when implementing Cas13-based technologies in bacteria and in eukaryotic cells. In bacteria, Cas13 was previously thought to induce widespread RNA cleavage as long as the target transcript was present (Abudayyeh et al., 2016). In contrast, in eukaryotic cells, Cas13 was reported to function as a sequence-specific gene silencer with no obvious off-target effects (Abudayyeh et al., 2017; Huynh et al.; Konermann et al., 2018; Mahas et al., 2019). To resolve this clear discrepancy, Cas13 was proposed to function differently in bacteria and in eukaryotic cells. At the same time, there are emerging reports of Cas13 inducing collateral RNA cleavage in

368 human cells, although cell-type specific factors were suggested as the underlying cause (Özcan 369 et al., 2021; Wang et al., 2019; Wu et al., 2021). The target expression threshold helps unify these 370 observations. In bacteria, target transcripts under threshold do not induce widespread collateral 371 RNA cleavage and instead are unaffected by targeting or undergo silencing. Applying our 372 proposed biochemical mechanism to eukaryotic cells, activated Cas 13 complexes would be much 373 less concentrated given the larger size of the cytoplasm and the distribution of transcripts in this 
374 cellular compartment that lend to a higher expression threshold. The few examples of collateral

375 cleavage might represent scenarios where the concentration of activated Cas 13 complexes or

376 the target transcript is much higher or localized close to important cellular RNAs. Investigating

377 Cas13 nucleases that exhibit less collateral activity (Abudayyeh et al., 2017; Xu et al., 2021) could

378 pose a simple solution to avoid potentially catastrophic RNA cleavage. The target expression

379 threshold therefore may impact not only bacterial immune defense but also the application of

380 Cas13 for programmable gene silencing in eukaryotic cells as well as for antivirals (Abbott et al.,

381 2020; Abudayyeh et al., 2017; Konermann et al., 2018).

383 STAR METHODS

\section{Strains and plasmids}

385 The strains and plasmids used in this study are listed in Table S2. The main plasmids used are pZ003 (LshCas13a), pFT50 (crRNA backbone), pFT50-repeat (crRNA backbone with repeat) and

387 pFT62 (target backbone). Spacers were inserted into the backbone by digestion with BsmBI and 388 ligation with Instant sticky-end ligase master mix (NEB, M0370S). Q5 mutagenesis or Gibson 389 assembly were used to modify the target plasmid or the nuclease backbone. All of the oligos used 390 in this research can be found in Table S3.

\section{Transformation-based targeting assays}

393 The transformation assays were conducted in two ways: targeting a genomically-encoded 394 transcript and a plasmid-encoded transcript. For genomically-encoded transcripts, biological 395 replicates containing the nuclease plasmid were inoculated overnight in LB medium (10 g 396 tryptone, $5 \mathrm{~g}$ yeast extract, and $10 \mathrm{~g} \mathrm{NaCl}$ in $1 \mathrm{~L}$ of $\left.\mathrm{dH}_{2} \mathrm{O}\right)$ with chloramphenicol $(\mathrm{Cm}, 34 \mu \mathrm{g} / \mathrm{mL})$. 397 After $16 \mathrm{~h}$, the $\mathrm{ABS}_{600}$ was measured and the samples were normalized, back-diluted 1:50 in fresh 398 LB with $\mathrm{Cm}$, and grown until an $\mathrm{ABS}_{600}$ of $0.6-0.8$. Cultures were placed on ice and made 399 electrocompetent by washing the pellet twice with $10 \%$ glycerol. Then, $50 \mathrm{ng}$ of the crRNA 
400 plasmids were transformed into $40 \mu \mathrm{L}$ of competent cells using the E. coli 1 program on the

401 MicroPulser Electroporator (Bio-rad). After $1 \mathrm{~h}$ of recovery in $500 \mu \mathrm{L}$ of SOC medium (SOB

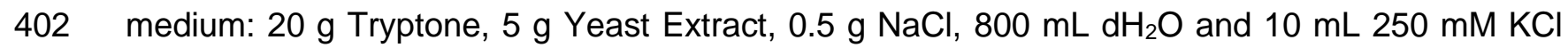

403 adjusted to $\mathrm{pH}$ 7. To SOB medium added $5 \mathrm{ml} 2 \mathrm{M} \mathrm{MgCl}_{2}, 20 \mathrm{ml}$ of $1 \mathrm{M}$ Glucose), 10 -fold dilutions

404 of the cultures in 1X PBS (10X PBS: $80 \mathrm{~g} \mathrm{NaCL}, 2 \mathrm{~g} \mathrm{KCl}, 17,7 \mathrm{~g} \mathrm{Na} 2 \mathrm{HPO}{ }^{\star} 2 \mathrm{H} 2 \mathrm{O}, 2.72 \mathrm{~g} \mathrm{KH}_{2} \mathrm{PO}_{4}$,

405 fill up to $1 \mathrm{~L}$ with $\mathrm{mqH} 2 \mathrm{O}$, set $\mathrm{pH}$ to 7.4 and autoclave) were prepared and $5 \mu \mathrm{L}$ spot dilutions

406 were plated on $\mathrm{Cm}$ and ampicillin (Amp, $100 \mu \mathrm{g} / \mathrm{mL}$ ) LB plates and incubated at $37^{\circ} \mathrm{C}$ for $16-18$

407 h.

408 For the plasmid-encoded transcripts, cultures containing the nuclease plasmid and the 409 target plasmid were transformed with the crRNA plasmid. The rest of the procedure matched that

410 followed for the genome targeting assay. The synthetic sequence expressed on a plasmid 411 (pFT62) contains part of the mRFP1 gene sequence, which does not match the E. coli genome.

412 Fragments from mRNAs (target plus 20 bp upstream and downstream) were cloned in pFT62 in

413 place of the synthetic target. Finally, to see if cloning an entire gene on a plasmid would change 414 the targeting outcome, full gene sequences with RBS and stop codon have been cloned in pFT62 415 through Gibson assembly.

\section{Flow cytometry analysis}

418 Plasmids expressing GFP under different Anderson promoters were cloned by Q5 mutagenesis 419 to introduce the different promoters in the pUA66-PJ23119 GFP plasmid. Cells containing the 420 nuclease plasmid and the GFP plasmid were inoculated overnight in LB medium with kanamycin 421 (Kan, $50 \mu \mathrm{g} / \mathrm{mL}$ ) and $\mathrm{Cm}$, then normalized, back-diluted to $\mathrm{ABS}_{600}=0.02$ and cultured until $\mathrm{ABS}_{600}$ $422 \approx 0.8$. Cells were then pelleted and resuspended in 1 X PBS before being applied to an Accuri C6 423 Plus analytical flow cytometer (BD Biosciences). 30,000 events were obtained by gating on living 424 cells, and the mean of the FL1-H values were quantified as GFP fluorescence. Final fluorescence 425 values were obtained by subtracting the autofluorescence of cells not expressing GFP. 


\section{L-arabinose induction of Cas13a-mediated targeting in E. coli}

428 The plasmid with the synthetic target under control of the arabinose-inducible promoter was

429 cloned by introducing the $\mathrm{P}_{\mathrm{BAD}}$ promoter in pFT62 through Gibson assembly. To validate this

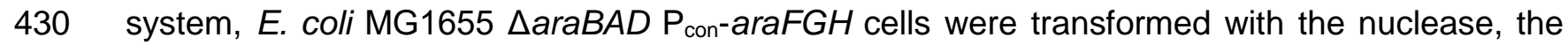

431 gRNA and the arabinose-inducible target plasmids. Three biological replicates were inoculated

432 overnight in LB supplemented with $\mathrm{Amp}, \mathrm{Cm}$, and Kan as well as $0.2 \%$ glucose to reduce

433 background expression from the $\mathrm{P}_{\mathrm{BAD}}$ promoter. The samples were then pelleted, resuspended

434 in LB with antibiotics and then back-diluted to $\mathrm{ABS}_{600}=0.01$ with or without $0.2 \%$ L-arabinose.

435 Culture turbidity was recorded over time on a Synergy Neo2 or $\mathrm{H} 1$ fluorescence microplate reader

436 (BioTek) for $16 \mathrm{~h}$ by measuring $\mathrm{ABS}_{600}$ every $3 \mathrm{~min}$.

\section{Assessment of collateral RNA cleavage}

439 Using the validated arabinose-inducible setup, collateral RNA cleavage was visualized on an 440 agarose gel. Cells with the nuclease, gRNA, and target plasmids were grown overnight in Cm, 441 Amp, Kan LB with $0.2 \%$ glucose, washed in LB with antibiotics to remove glucose, and back442 diluted to $\mathrm{ABS}_{600}=0.01$. The cells were cultured until $\mathrm{ABS}_{600} \approx 0.4$, after which each sample was 443 split in equal volumes with or without the inducer. After $1 \mathrm{~h}$ from induction, the $\mathrm{ABS}_{600}$ of each 444 sample was measured, and the same number of cells (2250 ABS ${ }_{600} \times \mathrm{mL}$ ) was snap frozen on dry 445 ice. The next day, total RNA was extracted using the Directzol RNA mini-prep plus kit (Zymo 446 research). Then $1 \mu \mathrm{g}$ of each RNA was mixed 2:3 with an RNA loading dye (2x) (for $50 \mathrm{~mL}: 625$ $447 \mu \mathrm{L}$ Bromophenol blue 2\%, $625 \mu \mathrm{L} 2 \%$ Xylene Cyanol, $1800 \mu \mathrm{L}$ of $0.5 \mathrm{M}$ EDTA pH = 8.0, 46,821 $448 \mathrm{~mL}$ Formamide), heated at $70^{\circ} \mathrm{C}$ for $10 \mathrm{~min}$, placed on ice, and resolved on a $1 \%$ TBE gel at 120 449 V for 40 min. RiboRuler High Range RNA ladder (Thermo Scientific, SM1821) was used as a size 450 marker. 


\section{Library design and validation}

453 The reference genome and annotation of E. coliK12 MG1655 (NC_000913.3) was used for gRNA

454 library design. First, all potential 32-nt guides were designed for protein-coding genes (limited to

455 the CDS) and rRNAs with non-GU PFS and GC content between $40 \%$ and $60 \%$, resulting in an

456 average of 484 guides per gene. To reduce the size of the library, and considering the unknown

457 effect of the targeting location within a gene on guide efficiency, each gene was divided into a

458 maximum of 10 sections with equal length. Within each section, guides were filtered based on the

459 strength of local secondary structure, defined as $\Delta G$, in both repeat-guide sequence and the

460 mRNA targeting region (including a region of 2 times length of $g R N A$ before and after the target).

$461 \Delta G$ was calculated as the energy difference between the unconstrained minimum free energy

462 (MFE) structure and the constrained MFE structure with no base pairs, estimated using RNAfold

463 from the Vienna RNA Package (Lorenz et al., 2011) version 2.4.12. Sequences (either guide or

464 flanking primer sequences) containing BsmBI restriction sites or homopolymer stretches of more

465 than four consecutive nucleotides were excluded to facilitate synthesis and cloning. The guide

466 with the lowest secondary structure strength in each section was selected, resulting in a library of

46725,997 guides, including 25,470 guides targeting protein-coding genes, 127 guides targeting

468 rRNAs, and 400 randomized non-targeting guides as negative controls. A sequence containing a

469 universal primer binding site and the BsmBI restriction site was added to the guides to amplify the

470 oligo library and digest it before ligating it into the backbone (see Table S4). The library was

471 synthesized by Twist Bioscience.

472 The base backbone pFT50 was slightly modified to insert the direct repeat before the GFP

473 dropout site to be able to limit the insertion size to the spacer itself. A BsmBI restriction site present

474 in pFT50 was also eliminated through Q5 mutagenesis.

475

476 Guide library cloning and verification 
477 The library was amplified with Kapa Hifi polymerase (20 ng DNA) for 10 cycles following the

478 manufacturer's instructions $\left(\mathrm{Ta}=64^{\circ} \mathrm{C} ; 30 \mathrm{~s}\right.$ denaturation, $20 \mathrm{~s}$ annealing, $15 \mathrm{~s}$ extension) using

479 primers SPCpr 349/350. $5 \mu \mathrm{L}$ library $(150 \mathrm{nM})$ and $5 \mu \mathrm{L}$ of backbone $(50 \mathrm{nM})$ were mixed in 25

$480 \mu \mathrm{L}$ of total reaction volume. The mixture was subjected to 50 cycles of BsmBI digestion ( 3 min at

$48142^{\circ} \mathrm{C}$ ) and ligation (T4 ligase $-5 \mathrm{~min}$ at $16^{\circ} \mathrm{C}$ ) with a final digestion at $55^{\circ} \mathrm{C}$ for 60 min to ensure

482 complete removal of the backbone, followed by a 10 minute heat inactivation at $80^{\circ} \mathrm{C}$. The sample

483 was then ethanol precipitated, and $5 \mu \mathrm{g}$ were transformed into fresh electrocompetent Top10 cells

$484(90 \mu \mathrm{L})$. The transformation was conducted with two separate batches of electrocompetent cells

485 to ensure enough transformants were obtained. After recovering the two cultures in $500 \mu \mathrm{L}$ of SOC

486 medium shaking at $37^{\circ} \mathrm{C}$ for $1 \mathrm{~h}$, the recovered cultures were back-diluted into $150 \mathrm{~mL} \mathrm{LB}$ with

487 Amp and cultured with shaking at $37^{\circ} \mathrm{C}$ for $12 \mathrm{~h}$. The next day, plasmid DNA from the culture was

488 isolated using the ZymoPURE II Maxiprep Kit (Zymo Research, D4203) and further purified by

489 ethanol precipitation.

491 Guide library screen

492 Two replicates of $E$. coli MG1655 cells with or without the nuclease plasmid were inoculated 493 overnight, then the next day the $\mathrm{ABS}_{600}$ was normalized and the cells back-diluted to $\mathrm{ABS} \mathrm{S}_{600} \approx 0.1$

494 in fresh LB with or without $\mathrm{Cm}$. Once each culture reached $\mathrm{ABS}_{600} \approx 0.8$, cells were made 495 electrocompetent by washing twice with $10 \%$ glycerol and finally resuspended in $480 \mu \mathrm{L} 10 \%$ 496 glycerol. For each sample, six separate transformations were conducted each with $1 \mu \mathrm{g}$ of library 497 DNA (40 $\mu \mathrm{L} /$ transformation). Transformed cells were then recovered in $500 \mu \mathrm{L}$ SOC medium for $4981 \mathrm{~h}$ with shaking at $37^{\circ} \mathrm{C}$. The six reactions were combined to yield $3 \mathrm{~mL}$ of culture per condition.

499 Serial dilutions of this culture were made, and $100 \mu \mathrm{L}$ of $1: 10,000$ dilutions were plated for the 500 targeting and no-Cas13a samples with the appropriate antibiotics ( $\mathrm{Cm}$ and Amp, or Amp only), 501 yielding a theoretical library coverage of $\sim 9,500$. The remaining culture was diluted 1:100 in LB 
502 with $\mathrm{Amp}$ and $\mathrm{Cm}$ to $\mathrm{ABS}_{600}=0.06$ and cultured for $12 \mathrm{~h}$ with shaking at $37^{\circ} \mathrm{C}$. Finally, the library

503 was isolated with the ZymoPure II Plasmid Midiprep Kit (Zymo Research, D4200).

\section{Next-generation sequencing of the guide libraries}

506 The guides sequences from the purified library DNA were amplified with Kapa Hifi polymerase 507 using primers oEV-315/316 (NT1), oEV-317/318 (NT2), oEV-319/320 (T1), oEV-321/322 (T2).

$50810 \mathrm{ng}$ of DNA were included in a 50- $\mu \mathrm{L}$ PCR reaction for 15 amplification cycles $\left(15 \mathrm{~s}\right.$ at $98^{\circ} \mathrm{C}, 30$

$509 \mathrm{~s}$ at $64^{\circ} \mathrm{C}, 30 \mathrm{~s}$ extension). The amplification products were purified using Ampure beads and 510 further amplified with primers oEV-323/324 (NT1), oEV-325/326 (NT2), oEV-327/328 (T1), oEV-

$511329 / 330$ (T2) to add the appropriate indices and Illumina adaptors. For this reaction, the same

512 settings were used with the only difference being the amount of input DNA (25 ng) and the number

513 of cycles (10). The resulting amplification products were purified with Ampure beads and resolved

514 on a gel to verify the presence of the correct amplicon. The samples were submitted for Sanger

515 sequencing and Bioanalyzer analysis as a quality check. Finally, samples were submitted for next-

516 generation sequencing at the NextSeq 500 sequencer (Illumina) with a 150 bp paired-ends kit

517 (130 million reads) to obtain 1000-fold coverage. To increase the library diversity, $20 \%$ of phiX 518 phage was spiked-in.

519 To correlate transcript expression levels with guide depletion, we measured transcript 520 levels in E. coli MG1655 under conditions paralleling the library screen. Briefly, two replicates of 521 cells containing the plasmid cBAD33 (empty backbone for nuclease plasmid) were cultured 522 overnight and then normalized to $\mathrm{ABS}_{600}=0.06$ in $\mathrm{LB}$ with $\mathrm{Cm}$ and cultured to $\mathrm{ABS}_{600} \approx 0.5$ or $523 \mathrm{ABS}_{600} \approx 0.8$. At those growth points, cells were pelleted and snap-frozen for RNA extraction with 524 the Directzol RNA mini-prep plus kit (Zymo Research, R2071). The samples were also DNase525 treated with TURBO DNase (Thermo Fisher Scientific, AM2239) and quality verified using a 526 Bioanalyzer 2100 (Agilent). Finally, rRNA was removed with the Rybo-off rRNA depletion kit 
527 (Vazyme Biotech, N407-01) and the samples were sequenced on a NovaSeq 6000 (Illumina) with

528 50-bp paired-end reads.

529 The resulting NGS data were deposited in NCBI's Gene Expression Omnibus (Edgar et

530 al., 2002) and are accessible through GEO Series accession number GSE179913 for the

531 genome-wide screen

532 (https://www.ncbi.nlm.nih.gov/geo/query/acc.cgi?acc=GSE179913)

533 and GSE179914 for transcriptomic analysis

534 (https://www.ncbi.nlm.nih.gov/geo/query/acc.cgi?acc=GSE179914).

536 Screen analysis and machine learning model

537 For analysis of the genome-wide screen, after merging using BBMerge (version 38.69) with 538 parameters "qtrim2=t, ecco, trimq=20, $-\mathrm{Xm \times 1g}, \mathrm{mix}=\mathrm{f}$ ", paired-end sequence reads with a perfect 539 match were assigned to gRNA sequences. After filtering guides for at least 1 count per million 540 reads in at least 2 samples, the library sizes were normalized using the read counts for non-

541 targeting guides with the trimmed mean of M-values (TMM) method in edgeR (Robinson and

542 Oshlack, 2010; Robinson et al., 2009) (version 3.28.0). Differential abundance of gRNAs between

543 targeting samples and control samples lacking the Cas13a nuclease was assessed using the 544 edgeR quasi-likelihood $\mathrm{F}$ test after fitting a generalized linear model. The translation initiation rate 545 of each gene was predicted using RBS calculator (version 1.0) (Salis, 2011).

546 For RNA seq analysis, sequencing reads were aligned to the E. coliK12 MG1655 genome 547 (NC_000913.3) using STAR (Dobin et al., 2013) (version 2.7.4a) with parameters “-548 alignIntronMax 1 --genomeSAindexNbases 10 --outSAMtype BAM SortedByCoordinate" and the 549 count of reads mapping to each gene was obtained using HTSeq (Anders et al., 2015) (version 550 0.9.1) with parameters “-i locus_tag -r pos --stranded reverse --nonunique none -t gene”, followed 551 by calculating transcripts per million (TPM). 
The machine learning regression model was developed with 144 features as predictors

553 and the log2FC values of gRNAs from the genome-wide screen as targets using auto-sklearn

554 version 0.10 .0 (Feurer et al., 2019) with all possible estimators and preprocessors included and

555 parameters 'ensemble_size': 1, 'resampling_strategy': 'cv', 'resampling_strategy_arguments':

$556\{$ 'folds': 5\}, 'per_run_time_limit': 360, and 'time_left_for_this_task': 3600. Features included gene

557 expression level (log2 transformed TPM at OD 0.4), gene essentiality, gene id, gene length,

558 (percent) targeting position in gene, delta G of repeat-gRNA and mRNA targeting region, and the

559 one-hot-encoded PFS sequence and gRNA sequence. Gene essentiality information in LB

560 Lennox medium was obtained from EcoCyc (Keseler et al., 2017) (https://ecocyc.org). The

561 optimal histogram-based gradient boosting model was evaluated using 10-fold cross-validation

562 and interpreted using TreeSHAP version 0.36.0 (Lundberg et al., 2020).

\section{Targeting assay with lower nuclease expression}

565 In order to look at targeting with varied nuclease expression, we used a plasmid expressing the

566 nuclease under the constitutive promoter PJ23108 (Pw). At first we assessed the relative

567 promoter strength of $\mathrm{Pw}$ and the native promoter $\left(\mathrm{P}_{\text {native }}\right)$ by cloning the gfp gene in the nuclease

568 backbone in place of the nuclease itself through Gibson assembly. Each gfp plasmid was

569 transformed with the target plasmid into E. coli MG1655 cells, and the 'Flow cytometry analysis'

570 protocol was followed to measure fluorescence of the two constructs. We then performed the

571 transformation-based targeting assay with the low expressed nuclease, the crRNA (T1) and

572 different Anderson promoters (P1 to P8) cloned in front of the synthetic target sequence.

\section{Cell-free transcription-translation assays}

575 Plasmids encoding the nuclease, a crRNA, a target sequence, and deGFP were used to assess

576 the targeting activity in a cell-free transcription-translation assay. The nuclease was either under

577 the control of $\mathrm{P}_{\text {native }}$ or Pw and the crRNA encoded either a targeting $(\mathrm{T})$ spacer or non-targeting 
578 (NT) control. To assess differences in targeting activity when the nuclease is under the control of

579 different promoters, the two nuclease constructs were added separately with either the targeting

580 or the non-targeting crRNA and the targeted plasmid to myTXTL Sigma 70 Master Mix (Arbor

581 Biosciences, 507005), with a final concentration of $2 \mathrm{nM}, 1 \mathrm{nM}$ and $0.5 \mathrm{nM}$, respectively. The

582 samples were incubated for $2 \mathrm{~h}$ at $29^{\circ} \mathrm{C}$, before a plasmid encoding deGFP was added to a final

583 concentration of $0.5 \mathrm{nM}$. The samples were then incubated at $29^{\circ} \mathrm{C}$ for $16 \mathrm{~h}$ in a plate reader

584 (BioTek Synergy Neo2) and fluorescence was measured every three minutes (excitation, 585 emission: $485 \mathrm{~nm}, 528 \mathrm{~nm}$ ). All shown data was produced using the Echo 525 Liquid Handler

586 (Beckman Coulter). The assays were therefore scaled down to $3 \mu$ reactions per replicate, with

587 four replicates each. As part of the analysis, the background fluorescence from myTXTL mix and

588 water samples was subtracted from all samples. Grubb's test was performed using the values

589 after $16 \mathrm{~h}$ to identify outliers between replicates $(\alpha=0.1)$. If no outliers were identified, the first of

590 the four replicates was discarded. The graph shows the average deGFP fluorescence over time

591 together with the standard deviation.

592

Infection experiments with MS2 phage

594 MS2 phage concentration $(\mathrm{PFU} / \mathrm{mL})$ has been calculated by performing a plaque assay.

595 Transcription-based targeting assays have been performed by transforming E. coli CGSC 4401

596 cells $(F+)$ expressing the nuclease and differentially abundant target rep or cp gene transcripts

597 with the correspondent targeting guides and by calculating the reduction in colony numbers

598 compared to a non-targeting guide. E. coli cells containing the active or dead nuclease and the

599 guide encoding plasmids have been grown overnight in selective LB medium, back-diluted to

$600 \mathrm{ABS}_{600}=0.05$ and let grow until $A B S_{600} \approx 0.3$. The samples have then been normalized to $A B S_{600}$

$601=0.3$ and aliquoted in a 96 well plate (Thermo Scientific, 167008) together with different amounts

602 of phages (MOI 0.1 and 5) or the respective volume of LB for the no-infection control. Cell growth

603 has been recorded over $16 \mathrm{~h}$ by measuring $\mathrm{ABS}_{600}$ every three minutes in a plate reader at $37^{\circ} \mathrm{C}$. 


\section{Tolerance to targeted $k a n R$ gene}

606 E. coli colonies containing the nuclease and crRNA $(\mathrm{K} 1, \mathrm{~K} 2)$ plasmids were transformed with the 607 targeted plasmid, which has a cloDF13 ori, different promoters in front of the kanR gene (P2, P5,

608 P8), and a non-targeted hygromycin (Hyg, $100 \mu \mathrm{g} / \mathrm{mL})$ resistance cassette. The plasmid was

609 cloned in two steps by Gibson assembly with pUA66 as the backbone. K1 and K2 were targeting

610 two different regions within the kanR transcript. The procedure is analogous to the transformation

611 assay with a plasmid-encoded transcript, with the targeted plasmid selected on Hyg. The next

612 day, colonies from the spot dilutions were counted, and colonies from the samples showing a

613 negligible reduction in transformation compared to the non-targeting control were inoculated

614 overnight in LB with $\mathrm{Cm}, \mathrm{Amp}$ and Hyg. Then the cultures were washed to remove Hyg and back-

615 diluted to $\mathrm{ABS}_{600}=0.01$ in either Hyg or Kan. The growth curves for the different conditions were

616 measured over time on the microplate reader for $14 \mathrm{~h}$ at $37^{\circ} \mathrm{C}$ by measuring $\mathrm{ABS}_{600}$ every $3 \mathrm{~min}$.

617 For generating the heatmap, $12 \mathrm{~h}$ time points were selected to compare the different conditions 618 and the resulting graph is the average of 9 biological replicates.

620 Data analysis and image visualization

621 Microsoft Excel was used to analyze the data, and GraphPad Prism was used to generate the bar 622 plots and heatmaps. The graphs were then modified in Adobe Illustrator to construct the final 623 figures. Transformation fold-reduction in the transformation assays was calculated as the ratio 624 between non-targeting and targeting colonies.

\section{Statistical analyses}

627 All statistical analyses were performed using a Welch's $t$ test assuming unequal variances. P628 values above 0.05 or average values lower than the reference average were considered non- 
629 significant. Statistical comparisons for the transformation assays relied on log values, which

630 assumes the samples are normally distributed on a log scale.

\section{ACKNOWLEDGMENTS}

633 We thank Fani Ttofali and Chunyu Liao for providing initial plasmids from which constructs used

634 in this work were generated. We also thank Tatjana Achmedov for technical advice and 635 assistance. This work was supported by the Joint Programming Initiative on Antimicrobial 636 Resistance (01KI1824 to C.L.B.), the National Institutes of Health (1R35GM119561 to C.L.B.), a 637 bayresq.net Bavarian research network grant (to L.B.), and the Defense Advanced Research

638 Projects Agency Safe Genes programme (HR0011-17-2-0042 to C.L.B.). The views, opinions 639 and/or findings expressed should not be interpreted as representing the official views or policies 640 of the Department of Defense or the U.S. Government.

\section{AUTHOR CONTRIBUTIONS}

643 Conceptualization: E.V., C.L.B.; Methodology: E.V., Y.Y., L.B., C.L.B.; Software: Y.Y., L.B.;

644 Validation: E.V., Y.Y., S.P.C., K.G.W.; Formal analysis: E.V., Y.Y., K.G.W., L.B.; Investigation:

645 E.V., S.P.C., K.G.W.; Writing - Original Draft: E.V., Y.Y., L.B., C.L.B.; Writing - Review and Editing:

646 E.V., Y.Y., S.P.C., K.G.W., L.B., C.L.B; Visualization: E.V., Y.Y., K.G.W., C.L.B.; Supervision:

647 L.B., C.L.B; Funding acquisition: L.B., C.L.B.

649 CONFLICT OF INTERESTS

650 C.L.B. is a co-founder and member of the Scientific Advisory Board for Locus Biosciences as well 651 as a member of the Scientific Advisory Board for Benson Hill. The other authors have no conflicts 652 of interest to declare.

\section{REFERENCES}


655

656

657

658

659

660

661

662

663

664

665

666

667

668

669

670

671

672

673

674

675

676

677

678

679

680

681

682

683

684

685

686

687

688

689

690

691

692

693

Abbott, T.R., Dhamdhere, G., Liu, Y., Lin, X., Goudy, L., Zeng, L., Chemparathy, A., Chmura, S., Heaton, N.S., Debs, R., et al. (2020). Development of CRISPR as an Antiviral Strategy to Combat SARS-CoV-2 and Influenza. Cell 181, 865-876.e12.

Abudayyeh, O.O., Gootenberg, J.S., Konermann, S., Joung, J., Slaymaker, I.M., Cox, D.B.T., Shmakov, S., Makarova, K.S., Semenova, E., Minakhin, L., et al. (2016). C2c2 is a singlecomponent programmable RNA-guided RNA-targeting CRISPR effector. Science 353, aaf5573.

Abudayyeh, O.O., Gootenberg, J.S., Essletzbichler, P., Han, S., Joung, J., Belanto, J.J., Verdine, V., Cox, D.B.T., Kellner, M.J., Regev, A., et al. (2017). RNA targeting with CRISPRCas13. Nature 550, 280-284.

Anders, S., Pyl, P.T., and Huber, W. (2015). HTSeq--a Python framework to work with highthroughput sequencing data. Bioinformatics 31, 166-169.

Barrangou, R., Fremaux, C., Deveau, H., Richards, M., Boyaval, P., Moineau, S., Romero, D.A., and Horvath, P. (2007). CRISPR provides acquired resistance against viruses in prokaryotes. Science 315, 1709-1712.

Bondy-Denomy, J., Pawluk, A., Maxwell, K.L., and Davidson, A.R. (2013). Bacteriophage genes that inactivate the CRISPR/Cas bacterial immune system. Nature 493, 429-432.

Borges, A.L., Zhang, J.Y., Rollins, M.F., Osuna, B.A., Wiedenheft, B., and Bondy-Denomy, J. (2018). Bacteriophage Cooperation Suppresses CRISPR-Cas3 and Cas9 Immunity. Cell 174, 917-925.e10.

Buchman, A.B., Brogan, D.J., Sun, R., Yang, T., Hsu, P.D., and Akbari, O.S. (2020).

Programmable RNA Targeting Using CasRx in Flies. CRISPR J 3, 164-176.

Charpentier, E., Richter, H., van der Oost, J., and White, M.F. (2015). Biogenesis pathways of RNA guides in archaeal and bacterial CRISPR-Cas adaptive immunity. FEMS Microbiol. Rev. $39,428-441$.

Cox, D.B.T., Gootenberg, J.S., Abudayyeh, O.O., Franklin, B., Kellner, M.J., Joung, J., and Zhang, F. (2017). RNA editing with CRISPR-Cas13. Science 358, 1019-1027.

Dobin, A., Davis, C.A., Schlesinger, F., Drenkow, J., Zaleski, C., Jha, S., Batut, P., Chaisson, M., and Gingeras, T.R. (2013). STAR: ultrafast universal RNA-seq aligner. Bioinformatics 29, 15-21.

East-Seletsky, A., O'Connell, M.R., Knight, S.C., Burstein, D., Cate, J.H.D., Tjian, R., and Doudna, J.A. (2016). Two distinct RNase activities of CRISPR-C2c2 enable guide-RNA processing and RNA detection. Nature 538, 270-273.

Elowitz, M.B., Levine, A.J., Siggia, E.D., and Swain, P.S. (2002). Stochastic Gene Expression in a Single Cell. Science 297, 1183-1186.

Feurer, M., Klein, A., Eggensperger, K., Springenberg, J.T., Blum, M., and Hutter, F. (2019).

Auto-sklearn: Efficient and Robust Automated Machine Learning. Automated Machine Learning 113-134.

Frost, L.S., Leplae, R., Summers, A.O., and Toussaint, A. (2005). Mobile genetic elements: the agents of open source evolution. Nat. Rev. Microbiol. 3, 722-732. 
694

695

696

697

698

699

700

701

702

703

704

705

706

707

708

709

710

711

712

713

714

715

716

717

718

719

720

721

722

723

724

725

726

727

728

729

730

731

732

Gasiunas, G., Barrangou, R., Horvath, P., and Siksnys, V. (2012). Cas9-crRNA ribonucleoprotein complex mediates specific DNA cleavage for adaptive immunity in bacteria. Proc. Natl. Acad. Sci. U. S. A. 109, E2579-E2586.

Goldberg, G.W., and Marraffini, L.A. (2015). Resistance and tolerance to foreign elements by prokaryotic immune systems - curating the genome. Nature Reviews Immunology 15, 717724.

Hampton, H.G., Watson, B.N.J., and Fineran, P.C. (2020). The arms race between bacteria and their phage foes. Nature $577,327-336$.

Hille, F., Richter, H., Wong, S.P., Bratovič, M., Ressel, S., and Charpentier, E. (2018). The Biology of CRISPR-Cas: Backward and Forward. Cell 172, 1239-1259.

Huynh, N., Depner, N., Larson, R., and King-Jones, K. A versatile toolkit for CRISPR-Cas13based RNA manipulation in Drosophila.

Jackson, S.A., McKenzie, R.E., Fagerlund, R.D., Kieper, S.N., Fineran, P.C., and Brouns, S.J.J. (2017). CRISPR-Cas: Adapting to change. Science 356.

Jinek, M., Chylinski, K., Fonfara, I., Hauer, M., Doudna, J.A., and Charpentier, E. (2012). A programmable dual-RNA-guided DNA endonuclease in adaptive bacterial immunity. Science 337, 816-821.

Kazlauskiene, M., Kostiuk, G., Venclovas, Č., Tamulaitis, G., and Siksnys, V. (2017). A cyclic oligonucleotide signaling pathway in type III CRISPR-Cas systems. Science 357, 605-609.

Keseler, I.M., Mackie, A., Santos-Zavaleta, A., Billington, R., Bonavides-Martínez, C., Caspi, R., Fulcher, C., Gama-Castro, S., Kothari, A., Krummenacker, M., et al. (2017). The EcoCyc database: reflecting new knowledge about Escherichia coli K-12. Nucleic Acids Res. 45, D543D550.

Kiga, K., Tan, X.-E., Ibarra-Chávez, R., Watanabe, S., Aiba, Y., Sato'o, Y., Li, F.-Y., Sasahara, T., Cui, B., Kawauchi, M., et al. (2020). Development of CRISPR-Cas13a-based antimicrobials capable of sequence-specific killing of target bacteria. Nat. Commun. 11, 2934.

Konermann, S., Lotfy, P., Brideau, N.J., Oki, J., Shokhirev, M.N., and Hsu, P.D. (2018). Transcriptome Engineering with RNA-Targeting Type VI-D CRISPR Effectors. Cell 173, 665676.e14.

Koonin, E.V., Makarova, K.S., Wolf, Y.I., and Krupovic, M. (2020). Evolutionary entanglement of mobile genetic elements and host defence systems: guns for hire. Nat. Rev. Genet. 21, 119131.

Landsberger, M., Gandon, S., Meaden, S., Rollie, C., Chevallereau, A., Chabas, H., Buckling, A., Westra, E.R., and van Houte, S. (2018). Anti-CRISPR Phages Cooperate to Overcome CRISPR-Cas Immunity. Cell 174, 908-916.e12.

Leenay, R.T., and Beisel, C.L. (2017). Deciphering, Communicating, and Engineering the CRISPR PAM. J. Mol. Biol. 429, 177-191.

Liao, C., Ttofali, F., Slotkowski, R.A., Denny, S.R., Cecil, T.D., Leenay, R.T., Keung, A.J., and Beisel, C.L. (2019). Modular one-pot assembly of CRISPR arrays enables library generation 
and reveals factors influencing crRNA biogenesis. Nat. Commun. 10, 2948.

734

Liu, L., Li, X., Wang, J., Wang, M., Chen, P., Yin, M., Li, J., Sheng, G., and Wang, Y. (2017a). Two Distant Catalytic Sites Are Responsible for C2c2 RNase Activities. Cell 168, 121-134.e12.

Liu, L., Li, X., Ma, J., Li, Z., You, L., Wang, J., Wang, M., Zhang, X., and Wang, Y. (2017b). The Molecular Architecture for RNA-Guided RNA Cleavage by Cas13a. Cell 170, 714-726.e10.

Lundberg, S.M., Erion, G., Chen, H., DeGrave, A., Prutkin, J.M., Nair, B., Katz, R., Himmelfarb, J., Bansal, N., and Lee, S.-I. (2020). From local explanations to global understanding with explainable AI for trees. Nature Machine Intelligence 2, 56-67.

Mahas, A., Aman, R., and Mahfouz, M. (2019). CRISPR-Cas13d mediates robust RNA virus interference in plants. Genome Biol. 20, 263.

Makarova, K.S., Wolf, Y.I., Alkhnbashi, O.S., Costa, F., Shah, S.A., Saunders, S.J., Barrangou, R., Brouns, S.J.J., Charpentier, E., Haft, D.H., et al. (2015). An updated evolutionary classification of CRISPR-Cas systems. Nat. Rev. Microbiol. 13, 722-736.

Makarova, K.S., Wolf, Y.I., Iranzo, J., Shmakov, S.A., Alkhnbashi, O.S., Brouns, S.J.J., Charpentier, E., Cheng, D., Haft, D.H., Horvath, P., et al. (2020). Evolutionary classification of CRISPR-Cas systems: a burst of class 2 and derived variants. Nat. Rev. Microbiol. 18, 67-83.

Marino, N.D., Pinilla-Redondo, R., Csörgő, B., and Bondy-Denomy, J. (2020). Anti-CRISPR protein applications: natural brakes for CRISPR-Cas technologies. Nature Methods 17, 471479.

Marraffini, L.A., and Sontheimer, E.J. (2010). CRISPR interference: RNA-directed adaptive immunity in bacteria and archaea. Nat. Rev. Genet. 11, 181-190.

Marshall, R., Maxwell, C.S., Collins, S.P., Jacobsen, T., Luo, M.L., Begemann, M.B., Gray, B.N., January, E., Singer, A., He, Y., et al. (2018). Rapid and Scalable Characterization of CRISPR Technologies Using an E. coli Cell-Free Transcription-Translation System. Mol. Cell 69, 146157.e3.

Marshall, R., Beisel, C.L., and Noireaux, V. (2020). Rapid Testing of CRISPR Nucleases and Guide RNAs in an Cell-Free Transcription-Translation System. STAR Protoc 1, 100003.

Meeske, A.J., and Marraffini, L.A. (2018). RNA Guide Complementarity Prevents Self-Targeting in Type VI CRISPR Systems. Mol. Cell 71, 791-801.e3.

Meeske, A.J., Nakandakari-Higa, S., and Marraffini, L.A. (2019). Cas13-induced cellular dormancy prevents the rise of CRISPR-resistant bacteriophage. Nature 570, 241-245.

Meeske, A.J., Jia, N., Cassel, A.K., Kozlova, A., Liao, J., Wiedmann, M., Patel, D.J., and Marraffini, L.A. (2020). A phage-encoded anti-CRISPR enables complete evasion of type VI-A CRISPR-Cas immunity. Science 369, 54-59.

Niewoehner, O., Garcia-Doval, C., Rostøl, J.T., Berk, C., Schwede, F., Bigler, L., Hall, J., Marraffini, L.A., and Jinek, M. (2017). Type III CRISPR-Cas systems produce cyclic oligoadenylate second messengers. Nature 548, 543-548.

van der Oost, J., Westra, E.R., Jackson, R.N., and Wiedenheft, B. (2014). Unravelling the 
structural and mechanistic basis of CRISPR-Cas systems. Nat. Rev. Microbiol. 12, 479-492.

772

773

774

775

776

777

778

779

780

781

782

783

784

785

786

787

788

789

790

791

792

793

794

795

796

797

798

799

800

801

802

803

804

805

806

807

808

Özcan, A., Krajeski, R., Ioannidi, E., Lee, B., Gardner, A., Makarova, K.S., Koonin, E.V., Abudayyeh, O.O., and Gootenberg, J.S. (2021). Programmable RNA targeting with the singleprotein CRISPR effector Cas7-11. Nature.

Partridge, S.R., Kwong, S.M., Firth, N., and Jensen, S.O. (2018). Mobile Genetic Elements Associated with Antimicrobial Resistance. Clinical Microbiology Reviews 31.

Ratner, H.K., Escalera-Maurer, A., Le Rhun, A., Jaggavarapu, S., Wozniak, J.E., Crispell, E.K., Charpentier, E., and Weiss, D.S. (2019). Catalytically Active Cas9 Mediates Transcriptional Interference to Facilitate Bacterial Virulence. Mol. Cell 75, 498-510.e5.

Robinson, M.D., and Oshlack, A. (2010). A scaling normalization method for differential expression analysis of RNA-seq data. Genome Biol. 11, R25.

Robinson, M.D., McCarthy, D.J., and Smyth, G.K. (2009). edgeR: A Bioconductor package for differential expression analysis of digital gene expression data. Bioinformatics 26, 139-140.

Rollie, C., Chevallereau, A., Watson, B.N.J., Chyou, T.-Y., Fradet, O., McLeod, I., Fineran, P.C., Brown, C.M., Gandon, S., and Westra, E.R. (2020). Targeting of temperate phages drives loss of type I CRISPR-Cas systems. Nature 578, 149-153.

Rostøl, J.T., and Marraffini, L.A. (2019). Non-specific degradation of transcripts promotes plasmid clearance during type III-A CRISPR-Cas immunity. Nat Microbiol 4, 656-662.

Salis, H.M. (2011). The ribosome binding site calculator. Methods Enzymol. 498, 19-42.

Smargon, A.A., Cox, D.B.T., Pyzocha, N.K., Zheng, K., Slaymaker, I.M., Gootenberg, J.S., Abudayyeh, O.A., Essletzbichler, P., Shmakov, S., Makarova, K.S., et al. (2017). Cas13b Is a Type VI-B CRISPR-Associated RNA-Guided RNase Differentially Regulated by Accessory Proteins Csx27 and Csx28. Mol. Cell 65, 618-630.e7.

Stern, A., Keren, L., Wurtzel, O., Amitai, G., and Sorek, R. (2010). Self-targeting by CRISPR: gene regulation or autoimmunity? Trends in Genetics 26, 335-340.

Theofilopoulos, A.N., Kono, D.H., and Baccala, R. (2017). The multiple pathways to autoimmunity. Nature Immunology 18, 716-724.

VanderWal, A.R., Park, J.-U., Polevoda, B., Kellogg, E.H., and O'Connell, M.R. (2021).

CRISPR-Csx28 forms a Cas13b-activated membrane pore required for robust CRISPR-Cas adaptive immunity.

Wang, B., Zhang, T., Yin, J., Yu, Y., Xu, W., Ding, J., Patel, D.J., and Yang, H. (2021).

Structural basis for self-cleavage prevention by tag:anti-tag pairing complementarity in type VI Cas 13 CRISPR systems. Mol. Cell 81, 1100-1115.e5.

Wang, Q., Liu, X., Zhou, J., Yang, C., Wang, G., Tan, Y., Wu, Y., Zhang, S., Yi, K., and Kang, C. (2019). The CRISPR- Cas13a Gene- Editing System Induces Collateral Cleavage of RNA in Glioma Cells. Advanced Science 6, 1901299.

Watanabe, S., Cui, B., Kiga, K., Aiba, Y., Tan, X.-E., Sato'o, Y., Kawauchi, M., Boonsiri, T., Thitiananpakorn, K., Taki, Y., et al. (2019). Composition and Diversity of CRISPR-Cas13a 
809 Systems in the Genus. Front. Microbiol. 10, 2838.

810 Wessels, H.-H., Méndez-Mancilla, A., Guo, X., Legut, M., Daniloski, Z., and Sanjana, N.E.

811 (2020). Massively parallel Cas13 screens reveal principles for guide RNA design. Nat.

812 Biotechnol. 38, 722-727.

813 Wu, Y., Jin, W., Wang, Q., Zhou, J., Wang, Y., Tan, Y., Cui, X., Tong, F., Yang, E., Wang, J., et 814 al. (2021). Precise editing of FGFR3-TACC3 fusion genes with CRISPR-Cas13a provides a 815 personalized therapeutic strategy for the treatment of human glioblastoma. Mol. Ther.

816 Xu, C., Zhou, Y., Xiao, Q., He, B., Geng, G., Wang, Z., Cao, B., Dong, X., Bai, W., Wang, Y., et 817 al. (2021). Programmable RNA editing with compact CRISPR-Cas13 systems from uncultivated 818 microbes. Nature Methods 18, 499-506. 


\section{FIGURE LEGENDS}

A

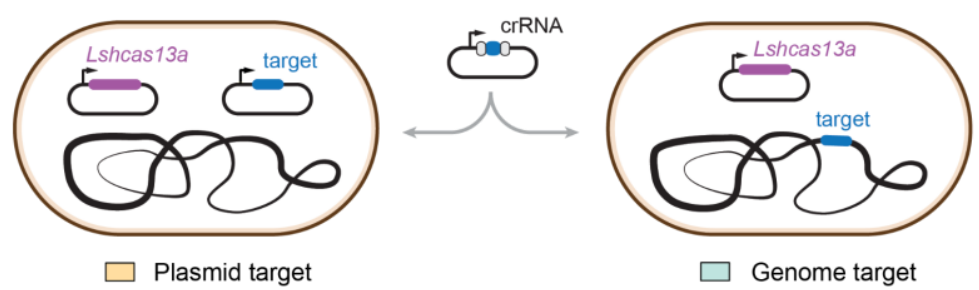

B

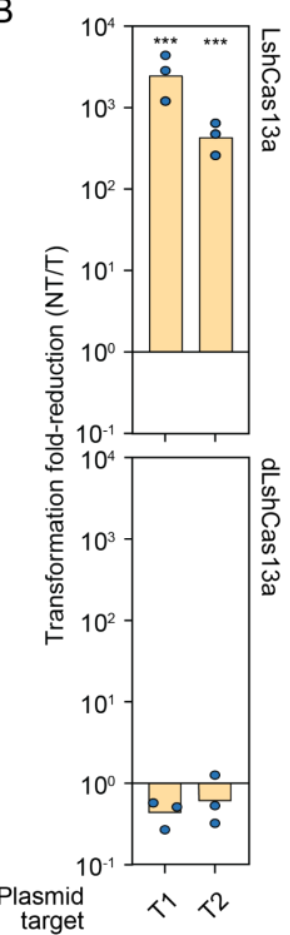

C

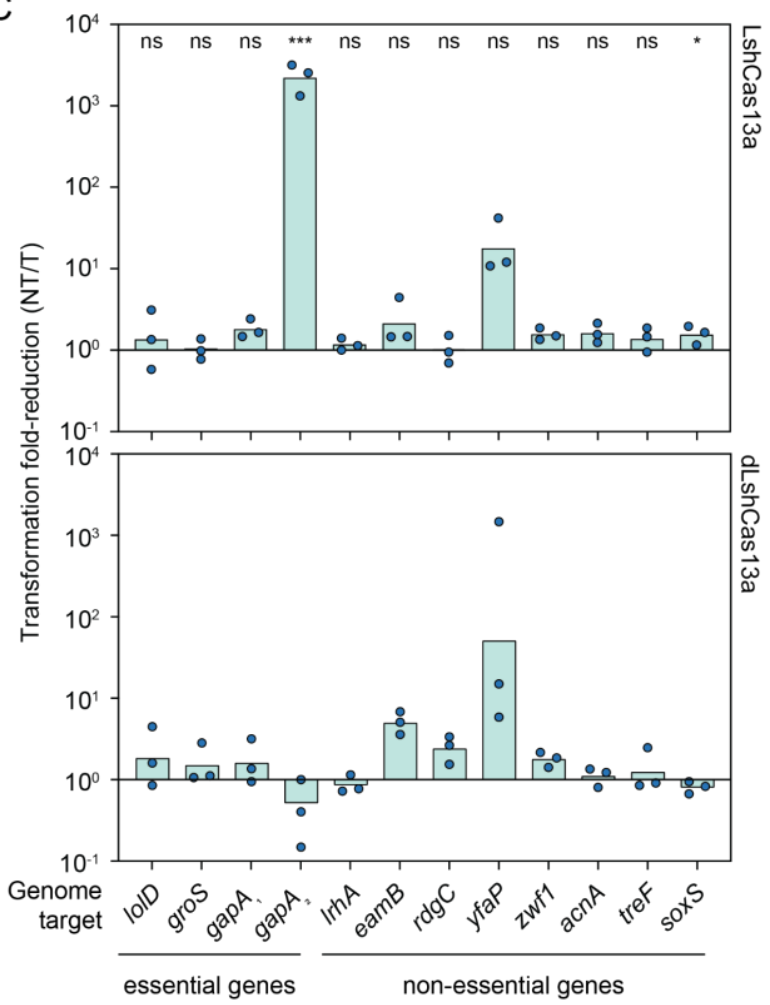

820 Figure 1. CRISPR-Cas13a is functional in E. coli yet fails to confer autoimmunity against selected

821 endogenous targets.

822 (A) Experimental setup for the plasmid transformation assay. A crRNA plasmid targeting either a

823 plasmid-encoded or genomically-encoded transcript is transformed into $E$. coli cells expressing

824 the L. shahii Cas13a (LshCas13a). The relative number of transformants compared to

825 transformation of a non-targeting crRNA plasmid are quantified.

826 (B) Impact of targeting a plasmid-encoded transcript with LshCas13a. dCas13a: catalytically827 dead Cas13a. Two different regions were targeted $(\mathrm{T} 1, \mathrm{~T} 2)$ within a transcript encoding a portion 828 of the mRFP1 gene. The transcript was expressed from the strong constitutive promoter P8. Fold- 
829 reduction was calculated as the ratio of transformants with the non-targeting (NT) and targeting

830 (T) crRNA plasmids.

831 (C) Impact of targeting different genomically-encoded transcripts with LshCas13a. Transcripts

832 were targeted from genes considered essential or non-essential in E. coli MG1655 under standard

833 growth conditions. gap $A_{1}$ and $g a p A_{2}$ represent two different target locations within the gapA

834 transcript.

835 Bars represent the mean of triplicate independent experiments. Statistical significance was 836 calculated by comparing the transformation fold-reduction for LshCas13a and dLshCas13a. ${ }^{* * *}$ :

$837 p<0.001 .^{* *}: p<0.01 .^{*}: p<0.05$. ns: not significant. 
A
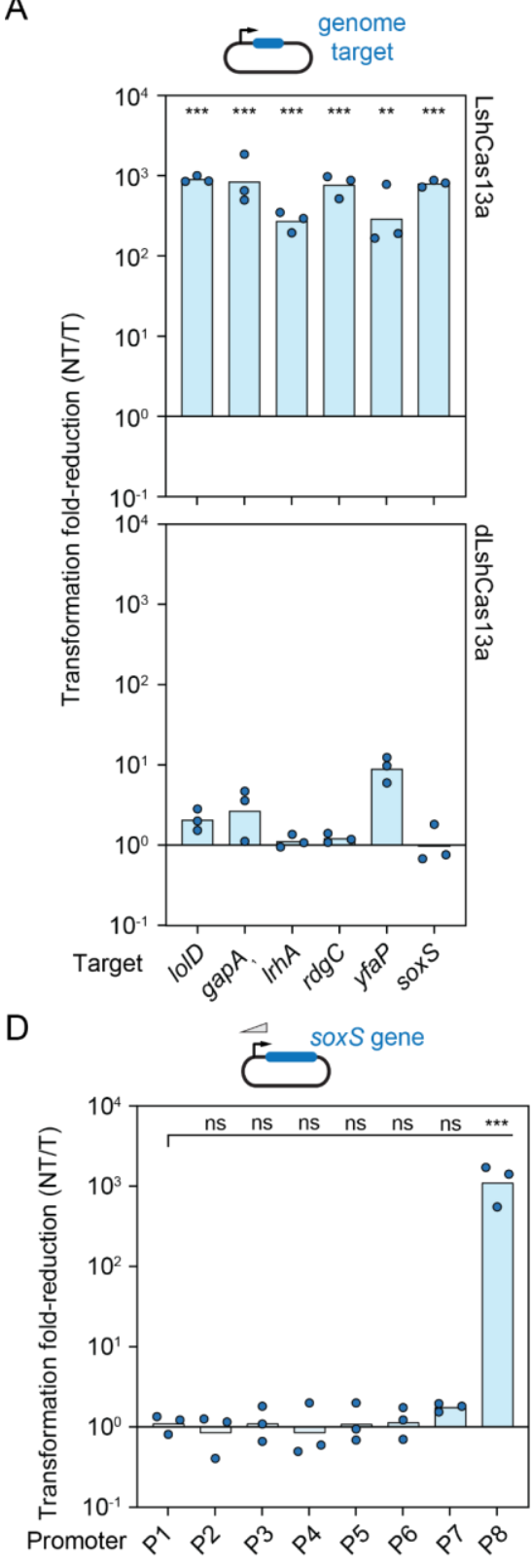

B

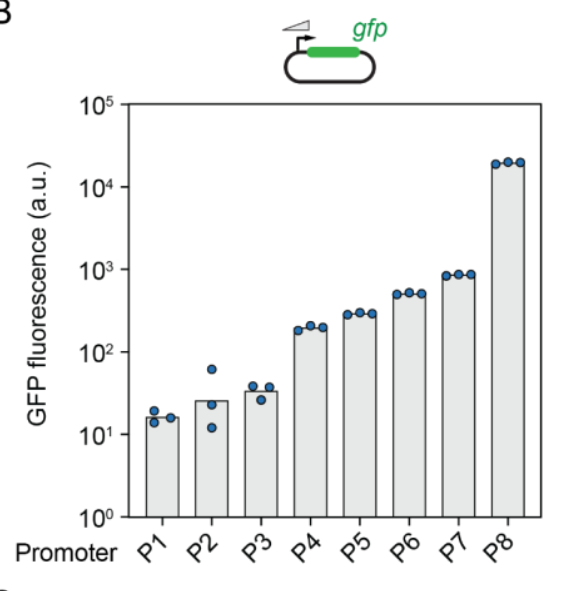

C

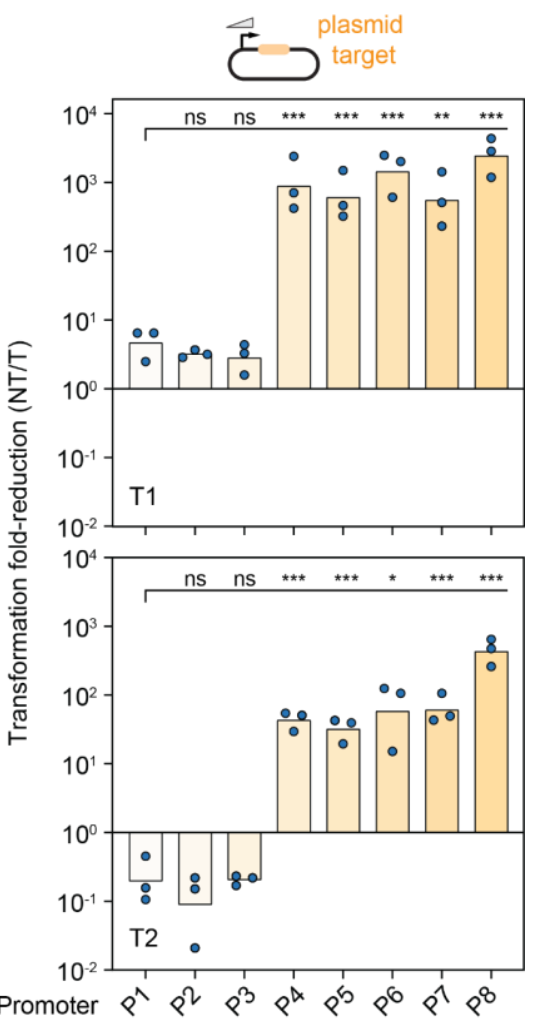

838 Figure 2. Cas13a-induced immunity requires target expression to exceed a threshold.

839 (A) Impact of over-expressing genomic targets in E. coli. Targets include the sequence

840 complementary to the crRNA guide along with the 20 nucleotides upstream and downstream and

841 are expressed under the strong constitutive promoter P8.

842 (B) Quantified strength of different constitutive promoters in E. coli. Promoter strength was 843 measured based on the fluorescence produced from a downstream gfp reporter. 
844 (C) Impact of targeting the plasmid-encoded transcript expressed from different constitutive

845 promoters by Cas13a in E. coli.

846 (D) Impact of targeting the plasmid-encoded soxS transcript expressed from different constitutive

847 promoters by Cas13a in E. coli. The genomic copy of soxS was intact in the E. coli strain.

848 Bars represent the mean of triplicate independent experiments. Statistical significance in A was

849 calculated by comparing the transformation fold-reduction for LshCas13a and dLshCas13a.

850 Statistical significance in $C$ and $D$ was calculated by comparing the transformation fold-reduction

851 to that of the weakest P1 promoter. ${ }^{* \star}: p<0.001{ }^{* *}: p<0.01 .^{*}: p<0.05$. ns: not significant. 
bioRxiv preprint doi: https://doi.org/10.1101/2021.11.23.469693; this version posted November 23, 2021. The copyright holder for this preprint (which was not certified by peer review) is the author/funder, who has granted bioRxiv a license to display the preprint in perpetuity. It is made available under aCC-BY-NC-ND 4.0 International license.

A

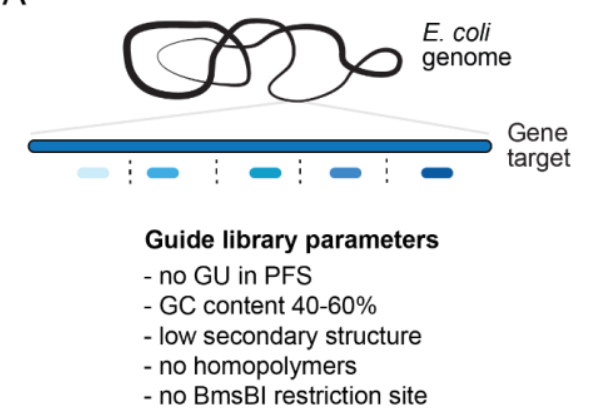

B

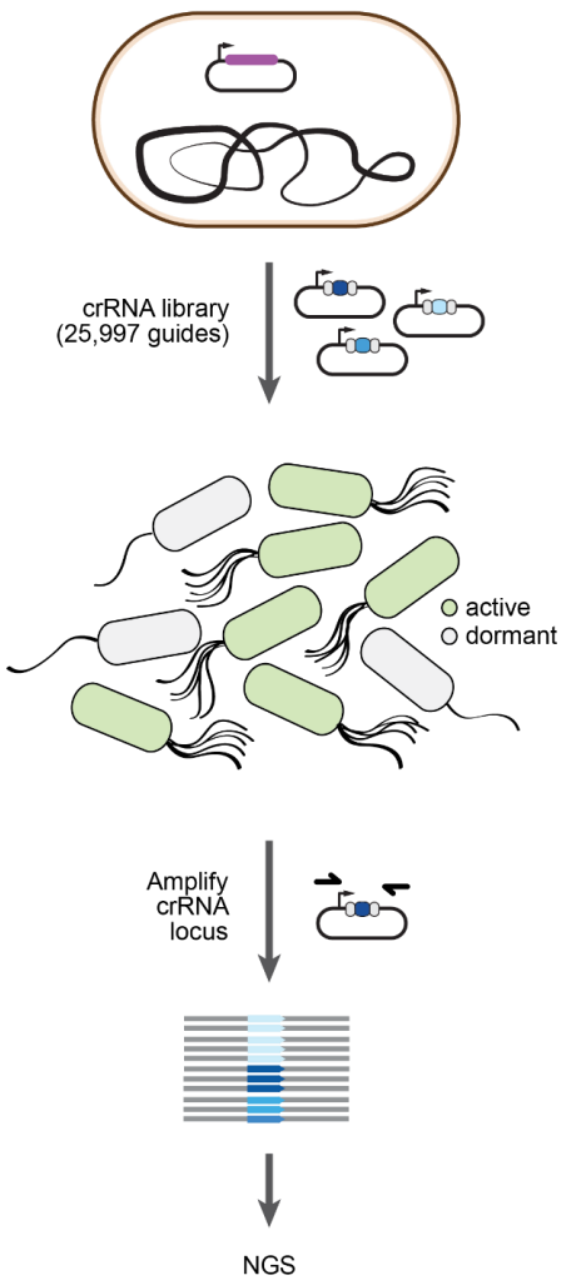

C

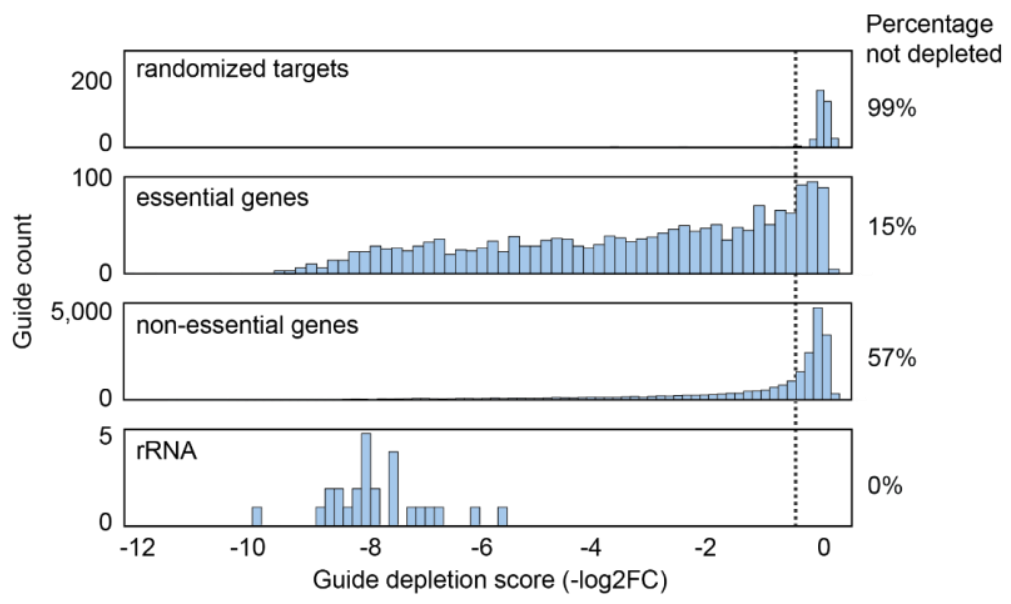

D
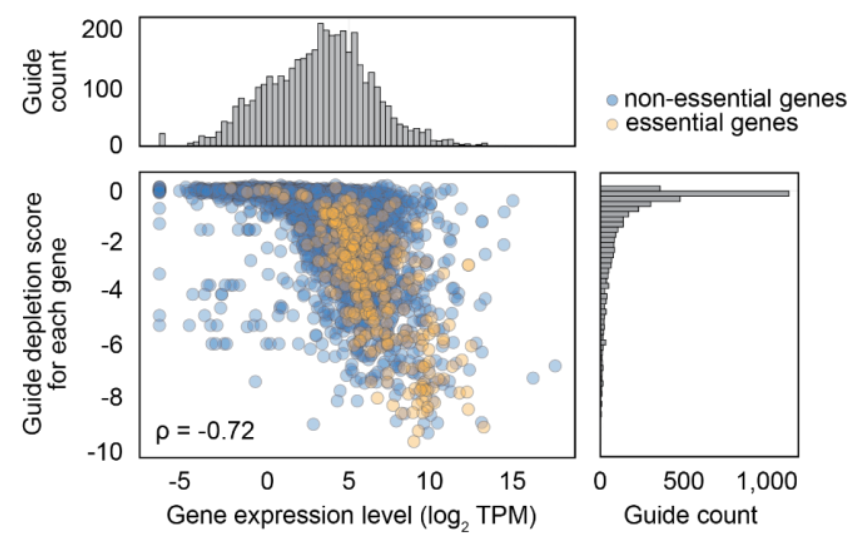

$\mathrm{E}$

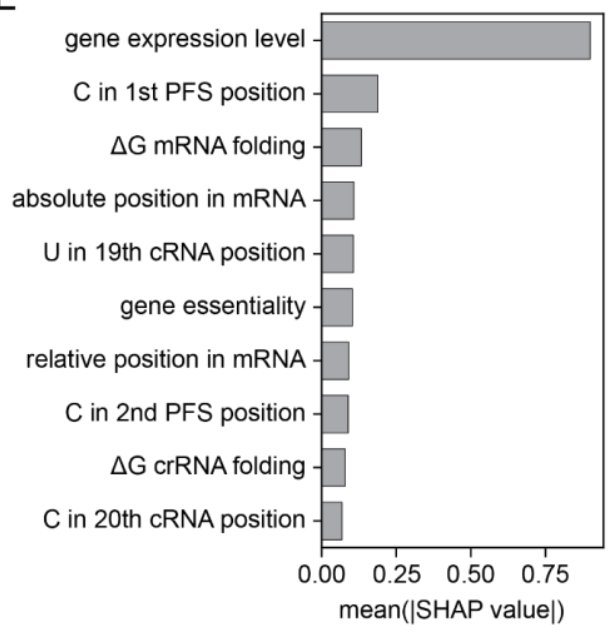

852 Figure 3. A genome-wide CRISPR-Cas13a screen reveals target expression levels as the main

853 determinant of cytotoxic self-targeting.

854 (A) Design of crRNA guide library. Guide selection accounted for standard rules lending to 855 efficient targeting and spanning the entire coding region of each target gene. Other parameters 
856 (i.e., homopolymers, BsmBI sites) were included to facilitate library synthesis and cloning. The

857 resulting library included 25,470 guides targeting protein-coding genes, 127 guides targeting

858 rRNAs, and 400 randomized guides as negative controls.

859 (B) Workflow for library screening. As part of the screen, cells with or without a LshCas13a

860 plasmid (purple) are transformed with the crRNA plasmid library and cultured while selecting all

861 present plasmids. Guide depletion is determined in comparison to the same workflow with the no-

862 LshCas13a control.

863 (C) Distribution of depletion scores for different groups of guides within the library. The cutoff for

864 no fitness defect is based on the range of depletion scores for a set of randomized guides.

865 (D) Correlation between guide depletion score and the expression levels of the target gene.

866 Expression levels were measured by RNA-seq analysis with $E$. coli cells harboring the no-

867 LshCas13a control and subjected to the library workflow to a turbidity of $A B S_{600} \approx 0.5$. Values for

868 transcript levels and guide depletion are the average of duplicate independent experiments and

869 screens, respectively. $\rho$ : Spearman coefficient. See Fig. S5A for the correlation for a turbidity of

$870 \quad \mathrm{ABS}_{600} \approx 0.8$.

871 (E) SHAP values for the strongest predictors of guide depletion from the library. The left barplot

872 indicates the average absolute contribution of each feature to the predicted depletion values,

873 while the right beeswarm plot shows the impact of each feature on each individual prediction. 

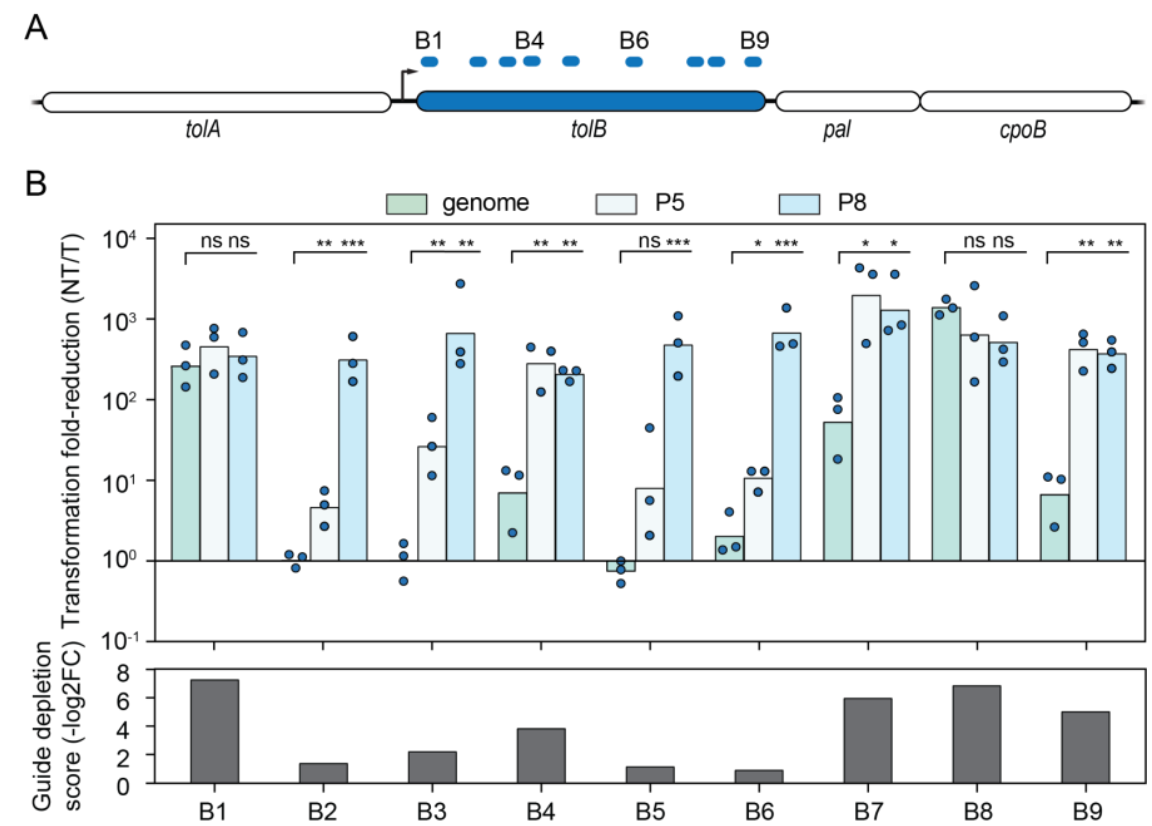

874 Figure 4. The target expression threshold varies between guide:target pairs associated with the 875 same transcript.

876 (A) Target locations within the tolB gene from the guide library. Targets are labeled B1 - B9.

877 (B) Impact of boosting tolB expression for each crRNA guide in $E$. coli. The tolB gene was 878 expressed from one of two constitutive promoters (P5 or P8) on a plasmid. The genomic copy of 879 tolB was always intact. Bottom: Average guide depletion score for each guide from the library 880 screen. Bars represent the mean of triplicate independent experiments. Statistical significance 881 was calculated by comparing the transformation fold-reduction to that with only the genomic copy 882 of tolB. ${ }^{* *}: p<0.001 .{ }^{* *}: p<0.01 .{ }^{*}: p<0.05$. ns: not significant. 


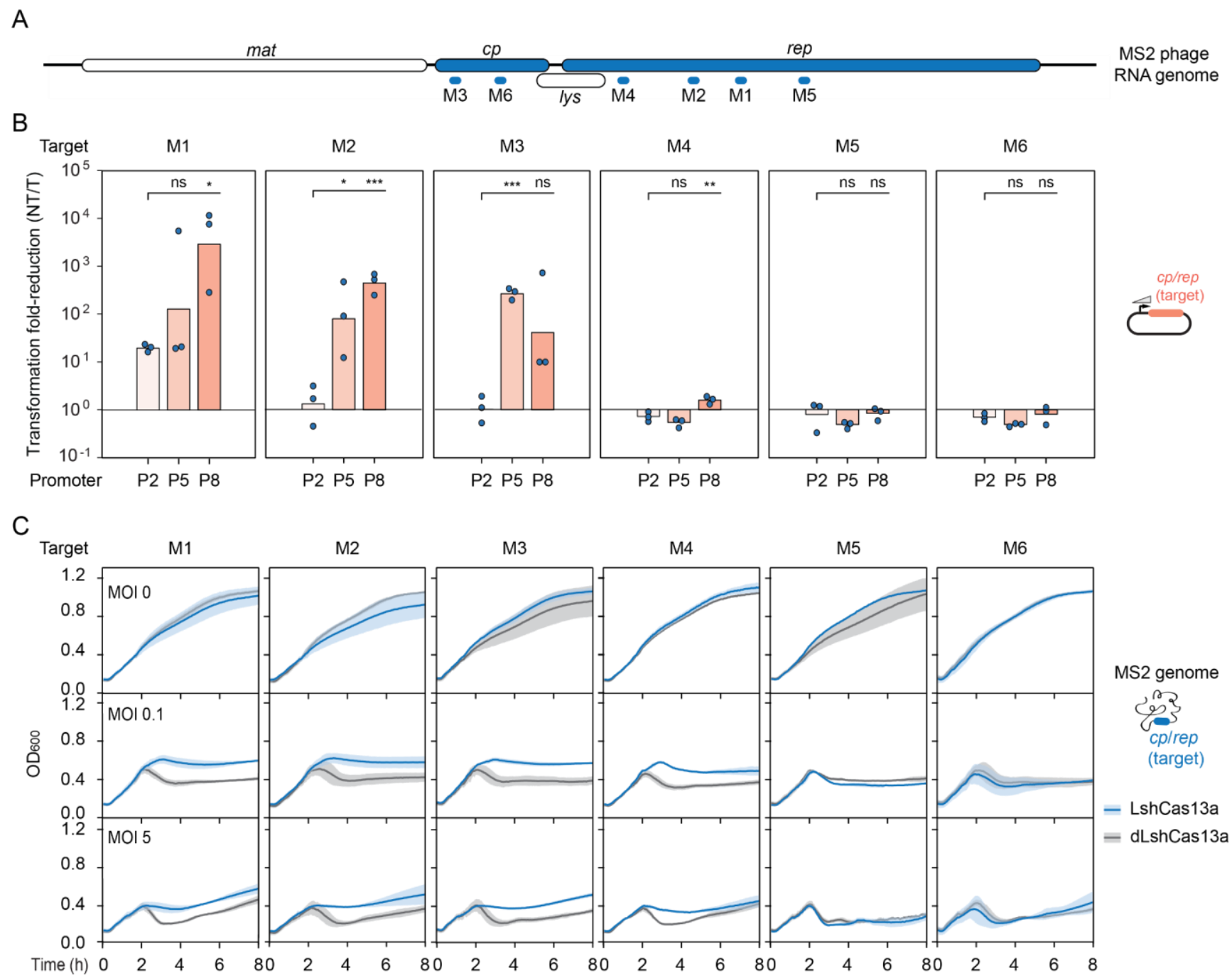

883 Figure 5. Cas13a defends E. coli from a lytic bacteriophage even with guides exhibiting a higher

884 immunity threshold.

885 (A) ssRNA genome of the lytic MS2 bacteriophage and location of guides targeting $c p$ and rep

886 genes used for accessing targeting and phage defense.

887 (B) Immunity threshold for different LshCas13a guides targeting cp or repgenes expressed under

888 different promoters on a plasmid. Bars represent the mean of triplicate experiments. Statistical

889 significance in B was calculated by comparing the transformation fold-reduction to that of the

890 weakest P2 promoter. ${ }^{* \star *}: p<0.001 .{ }^{* *}: p<0.01 .{ }^{*}: p<0.05$. ns: not significant or average below

891 the reference. 
bioRxiv preprint doi: https://doi.org/10.1101/2021.11.23.469693; this version posted November 23, 2021. The copyright holder for this preprint (which was not certified by peer review) is the author/funder, who has granted bioRxiv a license to display the preprint in perpetuity. It is made available under aCC-BY-NC-ND 4.0 International license.

892 (C) LshCas13a defense against MS2 bacteriophage at different MOls. Growth curves of E. coli 893 with active or dead LshCas13a are compared over time. The lines and bars represent respectively 894 the mean and standard deviation of three independent biological replicates. 
A

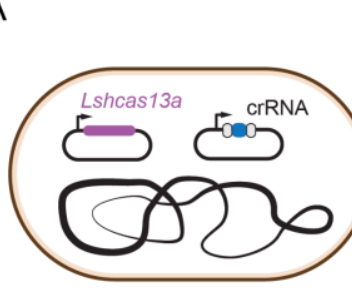

B

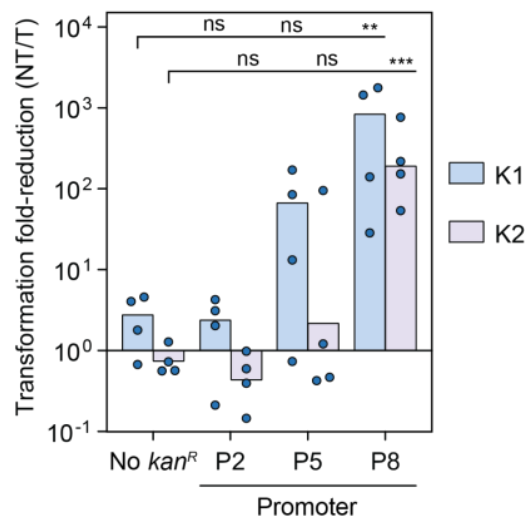

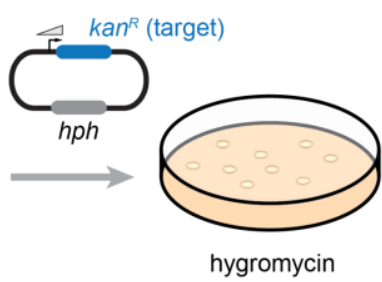

C
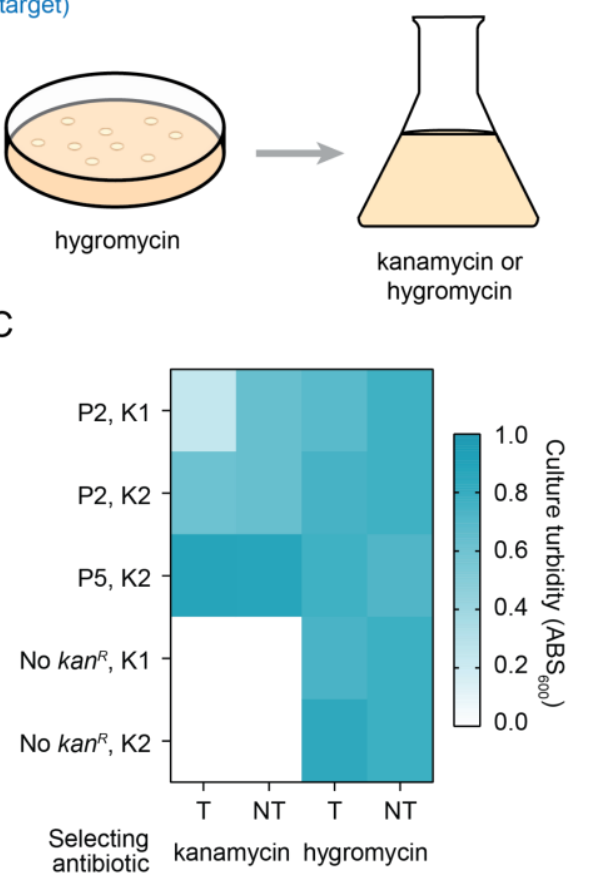

Figure 6. A plasmid transcript targeted by Cas13a and expressed under the threshold can be tolerated and can confer benefits to the host.

(A) Experimental setup for evaluating tolerance for a plasmid-expressed beneficial gene. The two targets $(\mathrm{K} 1, \mathrm{~K} 2)$ fall within the $\mathrm{kan}^{R}$ transcript, which is expressed under different constitutive promoters (P2, P5, P8).

(B) Impact of Cas13a-based targeting of the $\mathrm{kan}^{R}$ transcript expressed under the different constitutive promoters. Bars represent the mean of quadruplicate independent experiments. Statistical significance was calculated by comparing the transformation fold-reduction to that without the $\operatorname{kan}^{R}$ gene. ${ }^{* * *}: p<0.001 .{ }^{* *}: p<0.01 .{ }^{*}: p<0.05$. ns: not significant.

(C) Heat map comparing growth in the presence of kanamycin $(10 \mu \mathrm{g} / \mathrm{mL})$ or hygromycin $(100$ $\mu \mathrm{g} / \mathrm{mL}$ ) for constructs associated with a low transformation fold-reduction in B. Values represent the average turbidity after 12 hours of growth from 9 biological replicates. Constructs lacking the $\mathrm{kan}^{R}$ gene serve as negative controls, while the non-targeting crRNA plasmid serves as a nontargeting (NT) control. See Fig. S8 for the same growth measurements with a higher concentration of kanamycin $(50 \mu \mathrm{g} / \mathrm{mL})$. 


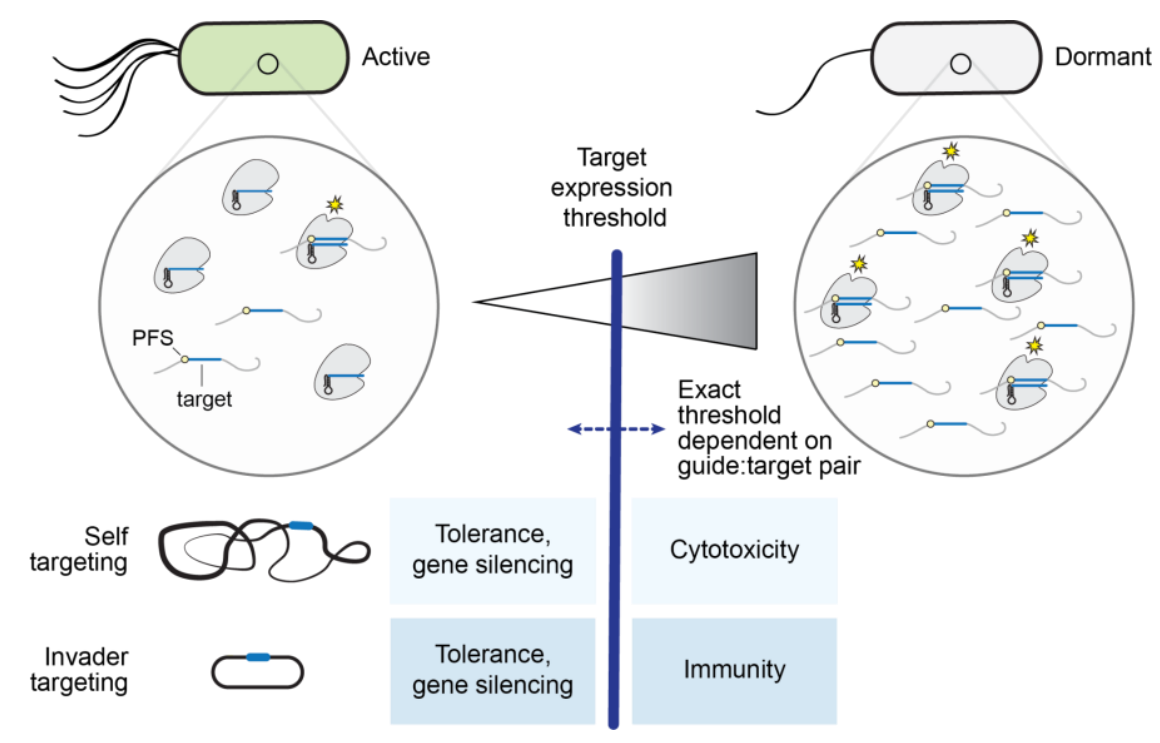

910 Figure 7. Proposed model for the target expression threshold as a determinant of Cas13-based

911 defense and autoimmunity. Any transcript with extensive complementarity to the crRNA guide

912 flanked by a PFS can be targeted by Cas13. However, the impact of targeting on the target gene

913 and the host cell depends on a third factor: a target expression threshold. Target expression under

914 the threshold is tolerated and can lead to targeted gene silencing, as supported by the extensive

915 depletion of guides targeting essential genes but not non-essential genes in the library screen

916 (Fig. 3) as well as reduced growth on kanamycin while targeting the $\mathrm{kan}^{R}$ resistance gene (Fig.

917 6C and S8). Target expression above the threshold leads to cytotoxicity for self-targeting and

918 immunity for invader targeting. The exact threshold is target-dependent, likely due to factors that

919 influence guide performance (e.g., local secondary structure, GC content). 